typeset using JPSJ.sty $<$ ver.1.0b $>$

\title{
Josephson-Vortex-Glass Transition in Strong Fields
}

\author{
Ryusuke Ikeda and Hiroto Adachi \\ Department of Physics, Kyoto University, Kyoto 606-8502
}

(Received March 29, 2000)

\begin{abstract}
A vortex-glass transition due to point disorder in layered superconductors is studied for the case with an applied field parallel to the layers. Our calculation of tilt responses indicates that, irrespective of the magnitude of the field, the resulting glass phase, Josephson-vortex-glass (JG), should have a transverse Meissner effect, as in a planar splayed glass phase, only for a tilt perpendicular to the layers. Further, focusing on the high field (and/or high anisotropy) region $B \sqrt{\Gamma}>\phi_{0} / d^{2}$, where $\Gamma$ is the mass anisotropy in the Lawrence-Doniach model, the JG transition line $T_{J G}(B)$ is shown to have a similar form to a $B-T$ line following from the disorder-free Lindemann criterion and to decrease with increasing $B \sqrt{\Gamma}$, in marked contrast to the disorder-free melting line insensitive to $B \sqrt{\Gamma}$ in such the high field region. This $T_{J G}(B)$ line seems to have been recently observed in a.c. susceptibility and in-plane resistivity measurements in BSCCO and qualitatively explains a field dependence at lower temperatures of previous BSCCO resistivity data showing the so-called in-plane Lorentz force-free behavior.
\end{abstract}

KEYWORDS: Type II superconductors, vortex states, Josephson vortex, vortex-glass

\section{$\S 1$. Introduction}

In bulk type II superconductors, different types of superconducting glass phases with no Ohmic dissipation are expected, depending on types and configurations of the static disorder (randomness), to be realized in a magnetic field. At least in homogeneous materials (i.e., with no granularity), no glass ordering is expected in zero field without transition to the Meissner phase because there is no origin of frustration. By contrast, in a nonzero field a spatial variation of $|\psi|$ due to fieldinduced vortices makes a formation of a glass phase with vanishing resistivity rather favorable at a low enough temperature.2.2.3) In this sense, the superconducting glass phases are peculiar to the nonzero field case and hence, can be called the vortex-glass phases. 16, (-)

So far, Ginzburg-Landau (GL) studiest, 1 ) of the vortex-glass transitions in a layered superconductor were limited to the most familiar case $(\mathbf{B} \| \mathbf{c})$ with a field perpendicular to the layers. No GL description of the case $(\mathbf{B} \perp \mathbf{c})$ in a field parallel to the layers and with no disorder was available even semiquantitatively until recently, and it was first performed in ref.5 in an approximated but systematic manner focusing on the lowest Landau level (LLL) modes of the pair-field $\psi$ 
(superconducting order parameter). Since a systematic description of the glass transitions in B $\| \mathbf{c}$ is available at present based only on the LLL approach, it is natural to, based on the results in ref.5, extend our study of a vortex-glass transition to $\mathbf{B} \perp \mathbf{c}$ case. It is our purpose in this paper to perform this.

The present study was motivated in part by resistive data of high $T_{c}$ materials. First, the vanishing behaviors of Ohmic dissipation in real YBCO materials in $\mathbf{B} \perp \mathbf{c}$ are controversial. 6 , 0 , 8, (6) Resistive data 8 of $90 \mathrm{~K}$ YBCO for a current perpendicular to $\mathbf{B}$ and parallel to the layers have indicated a continuous thermodynamic transition, while the c-axis resistive $\left(\rho_{c}\right)$ data in ref.7 have shown a first order transition as well as in $\mathbf{B} \| \mathbf{c}$. More recently, a resistive measurement 9 ) in $\mathrm{YBa}_{2} \mathrm{Cu}_{4} \mathrm{O}_{8}$ has shown an enhancement of a sharp vanishing, suggestive of a thermodynamic first order transition, of $\rho_{c}$ with decreasing the tilt angle between the $\mathbf{B}$ direction and the $\mathrm{CuO}$ planes. Such a vanishing, at least, of $\rho_{c}$ in $\mathbf{B} \perp \mathbf{c}$ cannot be understood without point disorder on the $\mathrm{CuO}$ layers. 专) Further, since the vanishing of resistivities in vortex systems is generally of a collective nature, a collective disorder effect near a freezing transition must appear irrespective of the current direction. Clearly, these observations 6 , 目) need to be discussed in the context of a point-disorderinduced glass transition.

Second, to our knowledge, the issue of the so-called in-plane Lorentz force free behavior 1 ) in BSCCO in tesla range has not been resolved sufficiently. In tesla range of this material satisfying $B \gg \phi_{0} /\left(2 \pi \sqrt{\Gamma} d^{2}\right)$ (see $\left.\S 2\right)$, the resistive data are quantitatively independent of the relative angle between $\mathbf{B}$ and the applied current, both of which are applied in directions perpendicular to the $\mathbf{c}$ axis. This "force free" behavior was verified later in refs.11 and 12. To explain the data in ref.10, a (technically inevitable) deviation of the applied field from the $\mathrm{CuO}$ plane 13 ) and the presence of the so-called double-kink excitation 12 were argued in literatures. The former 13 interpretation ascribing the angular independence to a vortex flow of deviation-induced pancake vortices inevitably contradicts the tendency seen in Fig.2 of ref.11 that, at higher temperatures (around 86K) below $T_{c 0}$, the field dependence of resistivity disappears with increasing $B$. Further, the picture, used in ref.12, of a vortex liquid confined within the interlayer spacings with double-kinks thermallyexcited cannot 14 explain Ohmic dissipation seen 11, 12) in the disordered phase. In ref.15, one of us has argued based on the anisotropic GL fluctuation theory that, with no static disorder, the in-plane Lorentz force free behavior is due merely to a strong enough anisotropy and will simultaneously be accompanied by the absence of a $B$ dependence of resistive data in the same $B$ range. Although a statement similar to this remains valid in the Lawrence-Doniach (LD) model with layer structure inducing the intrinsic pinning effect (see §2) and explains the above-mentioned behavior ( $B$-independent "force free" behavior) in ref.11 at higher temperatures, the resistive data in Fig. 2 of refs.11 and 12 seem to show a $B$-dependence at lower temperatures with the "force free" phenomenon kept. Within the LD model, this $B$-dependence will be clarified only by taking 
account of point disorder on the $\mathrm{CuO}$ planes inevitably contributing at lower temperatures.

A vortex-glass transition temperature is determined as the temperature at which the uniform glass susceptibility $\chi_{G}(\mathbf{k}=0)$ diverges on cooling, where $\chi_{G}(\mathbf{k})$ is the Fourier transformation of the correlation function 16 )

$$
G_{G}\left(m d, \mathbf{R}_{\perp}\right)=d \sum_{j} \int \mathrm{d} y \mathrm{~d} z \overline{<\psi_{j}^{*}\left(\mathbf{r}_{\perp}\right) \psi_{j+m}\left(\mathbf{r}_{\perp}+\mathbf{R}_{\perp}\right)>\left.\right|^{2}}
$$

defined consistently with the Lawrence-Doniach model

$$
\begin{aligned}
& \mathcal{H}_{\mathrm{LD}}=d \sum_{j} \int \mathrm{d} y \mathrm{~d} z\left[\varepsilon_{0}\left|\psi_{j}\right|^{2}+\xi_{0}^{2} \sum_{\mu=y, z}\left|\left(-\mathrm{i} \partial_{\mu}+\frac{2 \pi}{\phi_{0}} A_{j, \mu}\right) \psi_{j}\right|^{2}+\Gamma^{-1}\left(\frac{\xi_{0}}{d}\right)^{2}\left|\psi_{j}-\psi_{j+1}\right|^{2}\right. \\
& \left.+\frac{b}{2}\left|\psi_{j}\right|^{4}\right] \\
& =\int \mathrm{d}^{3} r \sum_{m=-\infty}^{+\infty} \mathrm{e}^{\mathrm{i} 2 \pi m x / d}\left[\varepsilon_{0}|\psi(\mathbf{r})|^{2}+\xi_{0}^{2} \sum_{\mu=y, z}\left|\left(-\mathrm{i} \nabla_{\mu}+\frac{2 \pi}{\phi_{0}}\left(B x \hat{y}+\delta A_{\mu}(\mathbf{r})\right)\right) \psi(\mathbf{r})\right|^{2}\right. \\
& \left.+\Gamma^{-1}\left(\frac{\xi_{0}}{d}\right)^{2}|\psi(\mathbf{r})-\psi(\mathbf{r}+d \hat{x})|^{2}+\frac{b}{2}|\psi|^{4}\right],
\end{aligned}
$$

where $d$ is the spacing between the superconducting layers lying in $y$ - $z$ plane, $\psi_{j}\left(\mathbf{r}_{\perp}\right)$ with $\mathbf{r}_{\perp}=(y, z)$ in the first representation of eq.(1.2) is the pair-field (superconducting order parameter) on the $j$-th superconducting layer, $\varepsilon_{0} \simeq\left(T-T_{c 0}\right) / T_{c 0}, \Gamma$ the mass anisotropy, $\psi(\mathbf{r})$ a spatially continuous pairfield in the second representation of eq.(1.2), and $b \xi_{0}^{-2}$ is written, in terms of the zero temperature penetration depth $\lambda(0)$ or of the $2 \mathrm{D}$ Ginzburg number (i.e., fluctuation strength) $\varepsilon_{G}^{(2)}$, in the form $32 \pi^{3} \lambda^{2}(0) / \phi_{0}^{2}=2 \pi d \varepsilon_{G}^{(2)} / k_{\mathrm{B}} T_{c 0}$. Further, in the first representation we put $\mathbf{A}_{j}=B j d \hat{y}+\delta \mathbf{A}\left(j d, \mathbf{r}_{\perp}\right)$ by assuming $\mathbf{B} \| \hat{z} \perp \mathbf{c}(=\hat{x})$, where $\delta \mathbf{A}$ is an external gauge disturbance necessary in defining linear response quantities. In obtaining the second representation, the Poisson summation formula was merely used with no approximation. Hence, the Josephson vortices with no singular cores in the first representation are expressed, for convenience, in the second representation as singular vortices of $\psi(\mathbf{r})$. In ref.5, a field-tuned sequence of structural (mean field) transitions between various disorder-free vortex solids was examined in terms of the second representation.

Point disorder on the superconducting planes is introduced by adding, e.g., a random scalar potential term 17) to eq. (1.2)

$$
\mathcal{H}_{r p}=d \sum_{j} \int \mathrm{d} y \mathrm{~d} z u_{j}\left(\mathbf{r}_{\perp}\right)\left|\psi_{j}\left(\mathbf{r}_{\perp}\right)\right|^{2},
$$

where the random potential $u_{j}$ has zero mean and Gaussian ensemble $\overline{u_{j}\left(\mathbf{r}_{\perp 1}\right) u_{l}\left(\mathbf{r}_{\perp 2}\right)}=$ $\xi_{0}^{2} \Delta \delta^{(2)}\left(\mathbf{r}_{\perp 1}-\mathbf{r}_{\perp 2}\right) \delta_{j, l}$. Here we did not introduce other uncorrelated disorder terms because, as 
will be seen later, our main results in this paper are independent of the presence of such additional terms.

In $\S 2$, the model (1.2) with the term (1.3) is examined consistently with ref.5 using the LLL approximation, and the glass susceptibility $\chi_{G}(\mathbf{k})$ is examined to find a glass transition line of the Josephson vortex system, i.e., of $\mathbf{B} \perp \mathbf{c}$ case of the LD model by focusing on the nontrivial high field regime $B>\phi_{0} /\left(2 \pi d^{2} \sqrt{\Gamma}\right)$. In $\S 3$, it is demonstrated by examining tilt responses of the LD model and of the closely related uniaxially periodic mode 18 that the resulting Josephson-vortex-glass (JG) phase should have a transverse Meissner effect (TME) for a tilt perpendicular to the layers, while the response for a tilt within the layers (perpendicular to $\mathbf{c}$ axis) remains nonsuperconducting. As intuitively expected by identifying the superconducting layer in the present case with a well-

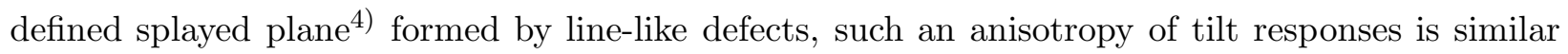
to that in the planar (Gaussian) splayed-glass phase examined in ref.4, although they are different in the origins of TME from each other. In $\S 4$, the resulting $\mathbf{B} \perp \mathbf{c}$ phase diagrams to be realized in a manner depending on the material parameters are discussed in details, and consistencies with available experimental data 11, 19) are pointed out.

\section{$\S 2 . \quad$ Glass Transition Line}

Let us begin with rewriting fl) $^{\text {) }}$ the LD model (1.2) (with no magnetic screening) by assuming the continuous pair-field $\psi(\mathbf{r})$ to be composed of the LLL modes. The disorder terms, eq.(1.3), will be omitted for the moment. We will express $\psi$ as $\psi(\mathbf{r})=\sum_{Q, q_{z}} \varphi\left(n, \mathbf{q}_{\perp}\right) u_{Q}(x, y) \exp \left(\mathrm{i} q_{z} z\right)$ with the LLL eigenfunction $u_{Q}(x, y) \propto \exp \left(\mathrm{i} Q y-\sqrt{\Gamma}\left(x+Q r_{B}^{2}\right) /\left(2 r_{B}^{2}\right)\right)$ in the gauge $\mathbf{A}=B x \hat{y}$ and substitute it into the second representation of eq.(1.2). Due to the layer structure with period $d$, the momentum $Q$ is written $E^{D}$ as $Q=r_{B}^{-2} d n+q_{y}$ with $r_{B}=\sqrt{\phi_{0} / 2 \pi B}$, where $\left|q_{y}\right|<d r_{B}^{-2} / 2$, and the partial LLL degeneracy is measured by the integer $n$ with the degree of degeneracy $N_{d} \equiv$ $L_{x} / d$, where $L_{\mu}$ denotes the linear system size in $\mu$-direction. For the moment, a constant gauge disturbance $\delta \mathbf{A}$ will be assumed. Using the Poisson summation formula, the quadratic part of eq.(1.2) is expressed as

$$
\begin{gathered}
\mathcal{H}_{2}=\xi_{0}^{2} \sum_{Q, q_{z}}\left(\xi_{0}^{-2} \varepsilon_{0}+\left\langle\left(\frac{2 \pi}{\phi_{0}} \delta A_{y}-q_{y}+\frac{d}{r_{B}^{2}} m\right)^{2}\right\rangle_{m}\right. \\
\left.+\frac{2}{\Gamma d^{2}}\left(1-\mathrm{e}^{-p / 4}<\exp \left(-p\left(m+1 / 2-q_{y} r_{B}^{2} / d\right)^{2}\right)>_{m}\right)+\left(\frac{2 \pi}{\phi_{0}} \delta A_{z}-q_{z}\right)^{2}\right)\left|\varphi\left(n, \mathbf{q}_{\perp}\right)\right|^{2}
\end{gathered}
$$

where

$$
p=\frac{\sqrt{\Gamma} d^{2}}{r_{B}^{2}}=\frac{2 \pi d^{2} \sqrt{\Gamma} B}{\phi_{0}}
$$

is a dimensioless field playing important roles in $\mathbf{B} \perp \mathbf{c}, \varphi\left(n, \mathbf{q}_{\perp}\right)$ was rescaled in the way $\varphi / \sqrt{h\left(q_{y}\right)} \rightarrow \varphi, h^{-1}\left(q_{y}\right)=\sum_{m} \exp \left(-p\left(m-q_{y} r_{B}^{2} / d\right)^{2}\right)$, and $<s(m)>_{m}$ denotes $h\left(q_{y}\right) \times$ 
$\sum_{m}\left[s(m) \exp \left(-p\left(m-q_{y} r_{B}^{2} / d\right)^{2}\right)\right]$. Assuming $\mathrm{e}^{-p / 2} \ll 1$ and neglecting higher order terms in $q_{y}$, eq.(2.1) is simplified in the form

$$
\begin{gathered}
\mathcal{H}_{2} \simeq \sum_{\mathbf{Q}}\left(\mu_{0}+\xi_{0}^{2}\left(q_{z}-\frac{2 \pi}{\phi_{0}} \delta A_{z}\right)^{2}\right. \\
\left.+\xi_{0}^{2}\left(q_{y}^{2}\left(1-2 e^{-p / 2}\right)-2 q_{y} \frac{2 \pi}{\phi_{0}} \delta A_{y}\left(1-4 p e^{-p}\right)+\left(\frac{2 \pi}{\phi_{0}} \delta A_{y}\right)^{2}\right)\right)\left|\varphi\left(n, \mathbf{q}_{\perp}\right)\right|^{2},
\end{gathered}
$$

where $\mu_{0} \simeq \varepsilon_{0}+2 \Gamma^{-1}\left(\xi_{0} / d\right)^{2}\left(1-2 \mathrm{e}^{-p / 2}\right)$. Hereafter, we assume the relation $\Gamma>2 \xi_{0}^{2} / d^{2}$, promising E $^{\text {) }}$ the presence of the $p>1$ region in which the vortices tend to be confined between two neighboring layers. Since only the LLL modes were taken into account here, the gauge field $\delta A_{y}$ in $x-y$ plane perpendicular to $\mathbf{B}$, as in $\mathbf{B} \| \mathbf{c}$ case, need not appear as the gauge-invariant combination $q_{y}-$ $2 \pi \delta A_{y} / \phi_{0}$ in $\mathcal{H}_{2}$. However, in the present $\mathbf{B} \perp \mathbf{c}$ case of the $\mathrm{LD}$ model, the $q_{y}^{2}\left|\varphi\left(n, \mathbf{q}_{\perp}\right)\right|^{2}$ and $q_{y} \delta A_{y}\left|\varphi\left(n, \mathbf{q}_{\perp}\right)\right|^{2}$ terms appear within LLL even for a smaller $p$ as a consequence of the partial breaking of the LLL degeneracy due to the layering. In particular, the latter term implies that the linear responses to $\delta A_{y}$, at least at low enough temperatures, can be defined within the LLL modes. Further, it is important to note that the last three terms of eq.(2.3) are summarized in the form $\xi_{0}^{2}\left(q_{y}-2 \pi \delta A_{y} / \phi_{0}\right)^{2}$, just as the second term of (2.3), if the $\mathrm{O}\left(\mathrm{e}^{-p / 2}\right)$ corrections in eq.(2.2) are neglected by assuming a strong field or a large enough anisotropy. This recovery of the isotropy in $y$ - $z$ plane in high $p$ should be expected in the LD model: Since, in this model, the pair-field is defined only on the discrete superconducting layers, the vortices in $p>1$ are confined between two neighboring layers, and consequently, the field-induced anisotropy on the layers tends to be lost with increasing $p$. This recovery of the $y-z$ isotropy and the fact 5 that the high field limit of the mean field transition line given by $\mu_{0}=0$ is the same as the exact result2d) suggest that the high field regime of the LD model may be well approximated by the present LLL approximation even in examining the response properties in $y-z$ plane. For these reasons, for later convenience, we will give the high field approximation of eq.(1.2) including the $|\psi|^{4}$ term in the form F $^{\text {) }}$

$$
\begin{aligned}
& \tilde{\mathcal{H}}_{\mathrm{LD}}(\varphi)= \sum_{\mathbf{Q}}\left(\mu_{0}+\xi_{0}^{2} \sum_{\mu=y, z}\left(q_{\mu}-\frac{2 \pi}{\phi_{0}} \delta A_{\mu}\right)^{2}\right)\left|\varphi\left(n, \mathbf{q}_{\perp}\right)\right|^{2}+\frac{b}{2 d L_{y} L_{z}} \\
& \times \sum_{\mathbf{Q}_{1}, \mathbf{Q}_{2}, \mathbf{Q}_{3}} V_{0}\left(n_{1}-n_{3}, n_{2}-n_{3}, q_{y, j}\right) \varphi^{*}\left(n_{1}, \mathbf{q}_{\perp, 1}\right) \varphi^{*}\left(n_{2}, \mathbf{q}_{\perp, 2}\right) \\
& \quad \times \varphi\left(n_{3}, \mathbf{q}_{\perp, 3}\right) \varphi\left(n_{1}+n_{2}-n_{3}, \mathbf{q}_{\perp, 1}+\mathbf{q}_{\perp, 2}-\mathbf{q}_{\perp, 3}\right)
\end{aligned}
$$

where

$$
\begin{gathered}
V_{0}\left(n_{1}-n_{3}, n_{2}-n_{3}, q_{y, j}\right)=\sqrt{h\left(q_{y, 1}+q_{y_{2}}-q_{y_{3}}\right) \prod_{j=1,2,3} h\left(q_{y, j}\right)} \\
\times \bar{v}\left(Q_{1}-Q_{3}\right) \bar{v}\left(Q_{2}-Q_{3}\right) \sum_{m} \bar{v}\left(Q_{1}+Q_{2}-2 d m / r_{B}^{2}\right)
\end{gathered}
$$


is a bare vertex function with $\bar{v}\left(Q_{i}\right)=\exp \left(-p\left(n_{i}+q_{y, i} r_{B}^{2} / d\right)^{2} / 2\right)$, and $\mathbf{Q}_{i}=Q_{i} \hat{y}+q_{z, i} \hat{z}=$ $\left(d n_{i} / r_{B}^{2}\right) \hat{y}+\mathbf{q}_{\perp, i}$.

The above analysis is not modified by replacing the constant $\delta \mathbf{A}$ assumed above with a $y$ dependent $\delta A_{z}$ and/or a $z$-dependent $\delta A_{y}$. This case is relevant to examining the tilt response near and above the glass transition and will be discussed in $\S 3$. Rather, we will comment here on the superconducting part $\sigma_{s, \mu \mu}(\mu=y$ or $z$ ) of dc (uniform) conductivities in the liquid region above $T_{m}$ where the pinning disorder term, eq.(1.3), may be neglected. According to the Kubo formula, it takes the form $\sigma_{s, \mu \mu}=-\partial \Upsilon_{\mu}(\mathbf{q}=0, i \Omega) /\left.\partial \Omega\right|_{\Omega \rightarrow+0}$, where $\Upsilon_{\mu}(\mathrm{i} \Omega)$ is a dynamical helicity modulus defined by substituting a $\tau$-dependent gauge disturbance $\delta A_{\mu}(\tau)$ into a quantum GL action $(\mathbf{l})$ consistent with eq.(1.2), $\tau$ the imaginary time, and $\Omega$ is an external Matsubara frequency. If the gauge field $\delta \mathbf{A}$ is spatially uniform, the derivation of a LLL quantum action with $\tau$-dependent $\delta \mathbf{A}$ is the same as the above derivation of eq.(2.4). Then, since the gradient and gauge-dependent terms when $e^{-p} \ll 1$ are isotropic in $y$ - $z$ plane, and the $\mathbf{q}_{\perp, l}$ dependence $(l=1,2$, or 3$)$ of the bare vertex function $V_{0}$ of eq.(2.4) is negligible, it is clear that the resulting $\sigma_{s, y y}$ and $\sigma_{s, z z}$ in such high fields are the same as each other, implying the in-plane "force-free" behavior mentioned in $\S 1$.

As is clear from the above derivation of eq.(2.4), this "force-free" behavior becomes more accurate with increasing $p=2 \pi B d^{2} \sqrt{\Gamma} / \phi_{0}$, i.e., with increasing $B$ and/or $\Gamma$. This is consistent with the experimental fact 12 that, in the same field range, the in-plane "force-free" behavior is seen not in less anisotropic YBCO but only in strongly anisotropic BSCCO. In the liquid regime in $p \gg 1$, the in-plane conductivities $\sigma_{s, \mu \mu}(\mu=y, z)$ are $B$-independent and well approximated by the fluctuation conductivity for $2 \mathrm{D}$ and $B=0$ case, obtained by neglecting the Kosterlitz-Thouless critical behavior, and have the form

$$
\sigma_{s, \mu \mu} \simeq \frac{\pi}{32 \mu_{c} R_{q} d} \exp \left(-\frac{2}{\varepsilon_{G}^{(2)}}\right) \exp \left(\frac{T_{c 0}}{\varepsilon_{G}^{(2)} T}\left(1-\frac{2 \xi_{0}^{2}}{d^{2} \Gamma}\right)\right),
$$

where $R_{q}=\pi \hbar / 2 e^{2}=6.45(\mathrm{k} \Omega)$ is the resistance quantum, $\mu_{c}$ is a constant of order unity, and a relation (2.21) (to be introduced later) was used here. Namely, the resistivity in the liquid regime in $p \gg 1$ yields the thermally activated behavior with no pinning effect.

In a previous paper, 15 ) one of us has ascribed the "force free" behavior to an extremely strong thermal fluctuation (equivalent to the vortex loop excitations) enhanced by the strong anisotropy of BSCCO, and has argued the discrete layer structure not to be essential to this phenomenon. The feature suggested in Fig. 2 of ref.11 that the $B$ dependence is lost with increasing $B$ indicates that the present explanation requiring the layer structure is more reasonable, and the assumption of an extremely strong fluctuation, which cannot be described within the LLL approximation, seems to be unnecessary in understanding this resistive behavior 111

We return to detailing our theory. The isotropic response in $y$ - $z$ plane mentioned above, i.e., the "force free" behavior does not change by including any point disorder unless this static disorder 
makes the supercurrent within the superconducting planes anisotropically random. Such a unusual possibility of disorder will not be considered here, and we will focus on eq.(1.3) as a reasonable model of such an isotropic point disorder. After replicating the model (1.2) with the term (1.3), the replicated effective hamiltonian21) becomes $\mathcal{H}_{\mathrm{eff}}=\sum_{a=1}^{n} \tilde{\mathcal{H}}_{\mathrm{LD}}\left(\varphi^{(a)}\right)+\sum_{a, b=1}^{n} \mathcal{H}_{\mathrm{ran}}\left(\varphi^{(a)}, \varphi^{(b)}\right)$, where

$$
\begin{gathered}
\mathcal{H}_{\mathrm{ran}}\left(\varphi^{(a)}, \varphi^{(b)}\right)=\frac{-\Delta^{(2)}}{2 \mathrm{k}_{\mathrm{B}} T d L_{y} L_{z}} \sum_{\mathbf{Q}_{1}, \mathbf{Q}_{2}, \mathbf{Q}_{3}} V_{0}\left(n_{1}-n_{3}, n_{2}-n_{3}, q_{y, j}\right) \\
\times \varphi^{(a) *}\left(n_{1}, \mathbf{q}_{\perp, 1}\right) \varphi^{(b) *}\left(n_{2}, \mathbf{q}_{\perp, 2}\right) \varphi^{(a)}\left(n_{3}, \mathbf{q}_{\perp, 3}\right) \varphi^{(b)}\left(n_{1}+n_{2}-n_{3}, \mathbf{q}_{\perp, 1}+\mathbf{q}_{\perp, 2}-\mathbf{q}_{\perp, 3}\right) .
\end{gathered}
$$

Using this replicated hamiltonian, the glass susceptibility within LLL consistent with $\mathcal{H}_{\text {eff }}$ is written, after using the Poisson summation formula, in the form

$$
\chi_{G}(\mathbf{k}) \simeq \mathcal{N} \sum_{\mathbf{Q}_{1}, \mathbf{Q}_{2}} e^{i k_{x} d\left(n_{1}-n_{2}\right)} \overline{<\varphi_{\mathbf{Q}_{1}} \varphi_{\mathbf{Q}_{2}}^{*}><\varphi_{\mathbf{Q}_{2}+\mathbf{k}_{\perp}} \varphi_{\mathbf{Q}_{1}+\mathbf{k}_{\perp}}^{*}>}
$$

where $\mathbf{k}_{\perp}=k_{y} \hat{y}+k_{z} \hat{z}$, and $\mathcal{N}$ is a normalization factor weakly dependent on $k_{y}$. In obtaining eq.(2.8), we assumed again $\mathrm{e}^{-p} \ll 1$.

Following the procedures used in B $\| \mathbf{c}$ case 1.22) let us consider the perturbation series of eq.(2.8), in particular its irreducible part $I_{\mathrm{ir}}$. In the limit of weak disorder, $I_{\mathrm{ir}}$ consists of a single impurity line, carrying a strength $\Delta$, with vertex corrections determined only by the interaction (quartic) term of eq.(2.4). Essential features to $I_{\text {ir }}$ can be seen in the study22, (1)) of $\chi_{G}(0)$ in the simplest 2 D limit of $\mathbf{B}\|\mathbf{c}\| \hat{z}$ which will be reviewed here. In this case, $I_{\text {ir }}$ is a constant so that $\chi_{G}(0)$ is the simple geometrical series, $\chi_{G}(0)=1+I_{\mathrm{ir}}+I_{\mathrm{ir}}^{2}+\cdots$. In a representation, corresponding to eq.(2.4), of hamiltonian in this $\mathbf{B} \| \mathbf{c}$ case, $I_{\text {ir }}$ obtained in ref.22 is expressed in the form

$$
\left.I_{\text {ir }}\right|_{2 \mathrm{~d}, \perp}=\frac{x_{R} \Delta_{\mathrm{eff}}}{2 \pi N_{v}^{1 / 2}} \sum_{k_{y}, p, p^{\prime}} v_{k_{y}} v_{p-p^{\prime}}\left(\delta_{p, 0}-2 x_{R} \bar{V}\left(k_{y} \mid p\right)\right)\left(\delta_{p^{\prime}, 0}-2 x_{R} \bar{V}\left(k_{y} \mid p^{\prime}\right)\right),
$$

where $\bar{V}\left(k_{y} \mid p\right)=N_{v}^{-1} \sum_{k_{x}} V_{\mathbf{k}} e^{-\mathrm{i} p k_{x} r_{B}^{2}}, \mathbf{k}$ is here a wave vector perpendicular to $\mathbf{B}, V_{\mathbf{k}}$ is a fully renormalized vertex part corresponding to the bare one $v_{k_{x}} v_{k_{y}}$ with $v_{k_{\mu}}=\exp \left(-k_{\mu}^{2} r_{B}^{2} / 2\right), \Delta_{\text {eff }}=$ $\Delta / \varepsilon_{G}^{(2)}(T)$, with $\varepsilon_{G}^{(2)}(T)=\varepsilon_{G}^{(2)} T / T_{c 0}$, is the effective pinning strength,, ,22) and $x_{R}$ is an interaction strength proportional to the squared pair-field propagators 1 According to the mean field result and a more general expression of the Abrikosov factor $\beta_{A}$, one noticest, 22) that, in the limit of the perfect (square) vortex lattice, $V_{\mathbf{k}_{\perp}}$ reduces to $\left(2 x_{R}\right)^{-1}\left(1-N_{v} \sum_{\mathbf{G} \neq=0} \delta_{\mathbf{k}, \mathbf{G}}\right)$, i.e.,

$$
\bar{V}\left(k_{y} \mid p\right) \rightarrow\left(2 x_{R}\right)^{-1}\left(\delta_{p, 0}+\delta_{k_{y}, 0}-\sum_{G_{2}} \delta_{k_{y}, G_{2}} \sum_{G_{1}} e^{-\mathrm{i} p G_{1} r_{B}^{2}}\right)
$$

where $\mathbf{G}=\left(G_{1}, G_{2}\right)$ is a reciprocal lattice vector, and $N_{v}$ the total number of vortices. Eq.(2.9) with eq.(2.10) simply becomes $\left.I_{\mathrm{ir}}\right|_{2 \mathrm{~d}, \perp} \propto N_{v}\left(\beta_{A}-1\right)$. Since the factor $\beta_{A}-1$ is always positive, reflecting a spatially varying $|\psi|$ in any vortex state, this prefactor $N_{v}$ in $I_{\text {ir }}$ indicates that, at the mean field level, a glass ordering occurs as soon as the vortices solidify on cooling. Namely, a 
spatial variation of $|\psi|$ (i.e., the fact $\beta_{A}>1$ ) in ordered phases, due to the presence of vortices, induces a glass ordering in nonzero fields contrary to in zero field case (see $\S 1$ ). In a more pinningdisordered case, the factor $N_{v}$ in the above relation is replaced with the number of vortices in a correlation area, $N_{\text {cor }}$, to be realized below a (if any) solidification transition (or crossover) line. This interpretation was justified22) noting the fact that a term in $V_{\mathbf{k}_{\perp}}$ corresponding to the last term of eq.(2.10) is nothing but the structure factor measuring the vortex-positional ordering. In a dirty enough case, the above-mentioned term in $V_{\mathbf{k}_{\perp}}$, just like above the melting line, becomes a continuous function of $\left|\mathbf{k}_{\perp}\right|$ decaying with increasing $\left|\mathbf{k}_{\perp}\right|$ even below the pinning-free melting line, and the positional correlation length shrinks to a value of the order of $r_{B}$ (Note that, as far as the fluctuation is weak or negligible, a nonzero positional correlation length is well-defined). Then, the factor $N_{\text {cor }}$ is found22 to decrease to a value of $\mathrm{O}(1)$, resulting in a decrease of the glass transition point. It is this situation with $N_{\text {cor }}$ of order unity which is accidentally described22) in the simplest ladder approximation with no 233) vertex corrections to the impurity line. Further, a decrease of temperature, i.e., an increase of $B$, along the melting line leads to an increase of $\Delta_{\text {eff }}$ and hence, corresponds to an enhancement of microscopic pinning. The $B-T$ phase diagram thus obtained 1 ) in $\mathbf{B} \| \mathbf{c}$ is consistent with recent data24) of twin-free YBCO (see $\S 4$ ). This example for $\mathbf{B} \| \mathbf{c}$ case indicates that an inclusion of a vertex correction, implying the vortex positional correlation, to the impurity line organizing $\chi_{G}$ is indispensable to understanding a field dependence of the glass transition temperature for a fixed pinning strength.

Now, to perform the corresponding analysis for the present $\mathbf{B} \perp \mathbf{c}$ case, let us first examine the fully-renormalized vertex part in the ideal limit of the perfect solid in $p>1$ which was called the $w=1$ regular solid in Ref.5. The mean field result of $\beta_{A}$ of this solid takes the forme

$$
\beta_{A}^{(1,1)}=\sum_{m, n}(-1)^{m n} V_{0}\left(m, n, q_{y, j}=0\right)
$$

In the cases including thermal fluctuations, $\beta_{A}$ is expressed as $f(0)+1$, where $f(\mathbf{R})$ is the densitydensity correlation function, 34 ) and takes the form

$$
\begin{gathered}
f(\mathbf{R})=N_{d}^{-1}\left(\sum_{n} e^{-p n^{2}} \sum_{\mathbf{q}_{\perp}} G\left(\mathbf{q}_{\perp}\right)\right)^{-2} \sum_{\mathbf{Q}_{1}, \mathbf{Q}_{2}, \mathbf{Q}_{3}} V_{0}\left(n_{1}-n_{3}, n_{2}-n_{3}-M, q_{y, j}=0\right) \\
\times\left(<\varphi^{*}\left(n_{1}, \mathbf{q}_{\perp, 1}\right) \varphi^{*}\left(n_{2}, \mathbf{q}_{\perp, 2}\right) \varphi\left(n_{3}, \mathbf{q}_{\perp, 3}\right) \varphi\left(n_{1}+n_{2}-n_{3}, \mathbf{q}_{\perp, 1}+\mathbf{q}_{\perp, 2}-\mathbf{q}_{\perp, 3}\right)>\right. \\
\left.-<\left|\varphi\left(n_{3}, \mathbf{q}_{\perp, 3}\right)\right|^{2}><\left|\varphi\left(n_{2}, \mathbf{q}_{\perp, 2}\right)\right|^{2}>\delta_{Q_{1}, Q_{3}}\right) e^{\mathrm{i}\left(Q_{3}-Q_{1}\right) Y},
\end{gathered}
$$

where $\mathbf{R}=(M d, Y)$ with an integer $M$. Noting that, due to the partial LLL degeneracy, 目) the fully-renormalized interaction term takes the form

$$
\begin{gathered}
\mathcal{H}_{4}^{\text {ren }}=\frac{b}{2 d L_{y} L_{z}} \sum_{\mathbf{Q}_{1}, \mathbf{Q}_{2}, \mathbf{Q}_{3}} V\left(n_{1}-n_{3}, n_{2}-n_{3}, \mathbf{q}_{\perp, j}\right) \varphi^{*}\left(n_{1}, \mathbf{q}_{\perp, 1}\right) \varphi^{*}\left(n_{2}, \mathbf{q}_{\perp, 2}\right) \varphi\left(n_{3}, \mathbf{q}_{\perp, 3}\right) \\
\times \varphi\left(n_{1}+n_{2}-n_{3}, \mathbf{q}_{\perp, 1}+\mathbf{q}_{\perp, 2}-\mathbf{q}_{\perp, 3}\right),
\end{gathered}
$$


and writing eq. $^{-}$(2.12) in terms of this renormalized vertex $V\left(m, n, \mathbf{q}_{\perp, j}\right)$ and comparing it with eq.(2.11), one finds that eq.(2.11) is recovered from eq.(2.12) when

$$
\frac{2 b}{d L_{y} L_{z}} \sum_{\mathbf{q}_{\perp}} G\left(\mathbf{q}_{\perp}\right) G\left(\mathbf{q}_{\perp}+\mathbf{k}_{\perp}\right) V\left(n_{1}, n_{2}, \mathbf{q}_{\perp}, \mathbf{q}_{\perp}^{\prime}, \mathbf{k}_{\perp}\right) \simeq(-1)^{1+n_{1} n_{2}}+\delta_{n_{1}, 0}+\delta_{n_{2}, 0},
$$

in the limit of vanishing $\mathbf{q}_{\perp}^{\prime}$ and $\mathbf{k}_{\perp}$. Notice that eq.(2.14) corresponds to eq.(2.10) in $\mathbf{B} \| \mathbf{c}$. By noting that the renormalized version of eq.(2.7) simply becomes

$$
\begin{gathered}
\mathcal{H}_{\mathrm{ran}}^{\mathrm{ren}}\left(\varphi^{(a)}, \varphi^{(b)}\right)=\frac{-\Delta^{(2)}}{2 \mathrm{k}_{B} T d L_{y} L_{z}} \sum_{\mathbf{Q}_{1}, \mathbf{Q}_{2}, \mathbf{Q}_{3}} \sum_{n_{4}, n_{5}} V_{0}\left(n_{1}-n_{3}, n_{4}-n_{5}, q_{y, j}\right) \\
\times C_{v}\left(n_{3}-n_{5}, n_{1}-n_{3}, \mathbf{q}_{\perp, 1}, \mathbf{q}_{\perp, 3}\right) C_{v}\left(n_{2}-n_{4}, n_{1}-n_{3}, \mathbf{q}_{\perp, 2}, \mathbf{q}_{\perp, 1}+\mathbf{q}_{\perp, 2}-\mathbf{q}_{\perp, 3}\right) \\
\times \varphi^{(a) *}\left(n_{1}, \mathbf{q}_{\perp, 1}\right) \varphi^{(b) *}\left(n_{2}, \mathbf{q}_{\perp, 2}\right) \varphi^{(a)}\left(n_{3}, \mathbf{q}_{\perp, 3}\right) \varphi^{(b)}\left(n_{1}+n_{2}-n_{3}, \mathbf{q}_{\perp, 1}+\mathbf{q}_{\perp, 2}-\mathbf{q}_{\perp, 3}\right),
\end{gathered}
$$

where

$$
C_{v}\left(n, m, \mathbf{q}_{\perp, 1}, \mathbf{q}_{\perp, 2}\right)=\delta_{n, 0}-\frac{2 b}{d L_{y} L_{z}} \sum_{\mathbf{q}_{\perp}} G\left(\mathbf{q}_{\perp}+\mathbf{q}_{\perp, 1}\right) G\left(\mathbf{q}_{\perp}+\mathbf{q}_{\perp, 2}\right) V\left(n, m, \mathbf{q}_{\perp}, \mathbf{q}_{\perp, 1}, \mathbf{q}_{\perp, 2}\right),
$$

and applying eq.(2.14) to this, the expression of eq.(2.15) simply becomes in clean limit

$$
\begin{gathered}
\mathcal{H}_{\mathrm{ran}}^{\mathrm{ren}}\left(\varphi_{\alpha}, \varphi_{\beta}\right)=\frac{-\Delta^{(2)}}{2 \mathrm{k}_{\mathrm{B}} T d L_{y} L_{z}} N_{d} \sum_{\mathbf{Q}_{1}, \mathbf{Q}_{2}, \mathbf{Q}_{3}}^{\left(n_{1} \neq n_{3}\right)}(-1)^{\left(n_{1}-n_{3}\right)\left(n_{2}-n_{3}\right)} \sum_{n} V_{0}\left(n_{1}-n_{3}, n, q_{y, j}=0\right) \\
\times(-1)^{n\left(n_{1}-n_{3}\right)} \varphi_{\alpha}^{*}\left(n_{1}, \mathbf{q}_{\perp, 1}\right) \varphi_{\beta}^{*}\left(n_{2}, \mathbf{q}_{\perp, 2}\right) \varphi_{\alpha}\left(n_{3}, \mathbf{q}_{\perp, 3}\right) \varphi_{\beta}\left(n_{1}+n_{2}-n_{3}, \mathbf{q}_{\perp, 1}+\mathbf{q}_{\perp, 2}-\mathbf{q}_{\perp, 3}\right) .
\end{gathered}
$$

The condition $n_{1} \neq n_{3}$, indicated above, in the summations of eq.(2.17) corresponds to a fact that the irreducible vertex $I_{\text {ir }}$ of $\chi_{G}$ is proportional to $\mathrm{e}^{-p}\left(\propto \beta_{A}-1\right)$ (see the expression of $r_{G}$ in eq.(2.19)) and implies that, as in $\mathbf{B} \| \mathbf{c}$, spatial variations of $|\psi|$ and of the supercurrent in a direction perpendicular to $\mathbf{B}$, which is the $y$-direction parallel to the superconducting planes in $\mathbf{B} \perp \mathbf{c}$, in the expected solid state are indispensable to a vortex glass ordering. The prefactor $N_{d}=L_{x} / d$ in eq.(2.17) corresponds to the prefactor $N_{v}$ in the resulting $\left.I\right|_{2 \mathrm{~d}, \perp}\left(\simeq N_{v}\left(\beta_{A}-1\right)\right)$ in $\mathbf{B} \| \mathbf{c}$ case and appeared here owing to the ideal assumption such that the vertex correction to the impurity line can be expressed with no pinning disorder. Due to this large factor, the glass transition in clean limit of $\mathbf{B} \perp \mathbf{c}$ case also occurs, as in $\mathbf{B} \| \mathbf{c}$ case, as soon as the vortices solidify at the disorder-free melting line $T_{m}(B)$.

Physically, the $N_{d}$-factor of eq.(2.17) implies the number in $x$-direction of Bragg spots, appearing below the melting line, at nonzero $k_{y}$ 's (As indicated in eq.(4.5) of ref.5, the "Bragg peaks" at zero $k_{y}$ already appear above the melting line, merely reflecting the original layer structure, and are unrelated to an ordering in the vortex state). To justify this identification, one has only to notice that the origin of this $N_{d}$-factor is a trivial (or, unlimited) summation of $n_{4}$ or $n_{5}$ in eq.(2.15) and that, in eq.(2.12), $\left(n_{2}-n_{3}\right) d$ plays, just like $n_{4} d$ and $n_{5} d$ in eq.(2.15), a role of a coordinate 
perpendicular to the layers, while $Q_{1}-Q_{3}$ is a wave vector in $y$-direction parallel to the layers there. Since a positional correlation length just below $T_{m}$ in real systems shrinks with effectively increasing disorder, the $N_{d}$-factor arising by assuming the limit of weak pinning should be replaced, in more realistic cases, with a $\Delta$-dependent smaller factor. Diagrammatically, this implies that, for such a highly disordered case, the impurity lines within the vertex correction to each impurity line organizing the ladder of $\chi_{G}$ are not negligible any longer. Although it is not easy to find a $\Delta$ dependence of the vertex correction within the framework of the present work, we expect by following the corresponding analysis in $\mathbf{B} \| \mathbf{c}$ case that, in such a disordered system, the first term of r.h.s. of eq.(2.14) will become a continuous function of $\left|n_{1}\right|$ and $\left|n_{2}\right|$ decaying with increasing $\left|n_{1}\right|$ or $\left|n_{2}\right|$ (This can be recognized through a perturbative computation valid above $T_{m}$ ). Then, the $n_{4}$ and $n_{5}$ summations in eq.(2.15) becomes convergent, and a prefactor in the corresponding one to eq.(2.17) does not depend on a system-size any longer. Hereafter, we will replace $N_{d}$ in eq.(2.17) with a smaller number $N_{c}(\Delta)$ by imagining a moderately disordered system. Similarly to that in B $\|$ c case, this $N_{c}$ physically corresponds to a (dimensioless) correlation length perpendicular to the layers. In such a moderately disordered system, the first order solidification transition at $T_{m}$ is weakened with increasing $p$ and without decreasing $T$ because the rigidity essential to the solidification, i.e., the shear rigidity, is exponentially small, S $^{\text {I }}$ and hence, the vortex system in higher $p$ is likely to be more susceptible 25) to the pinning disorder. Namely, besides that in $\mathbf{B} \| \mathbf{c}$ (see the preceding paragraph), this mechanism also contributes to disordering the solid due to an increase of $B$. Hence, the first order solidification line may terminate at a $p$-value $p_{c}$ before entering the $p \gg 1$ region. In a dirtier system with $p_{c}<1$, the layering effect in $p<p_{c}$ is a small correction fi $^{-}$ to the solidification, and thus, phenomena near $T_{m}$ in such $p$ 's will be similar to those in $\mathbf{B} \| \mathbf{c}$.

It is straightforward to derive an expression of $\chi_{G}(\mathbf{k})$ in terms of eq.(2.17), which takes the form

$$
\begin{aligned}
& \chi_{G}(\mathbf{k})=\int_{\mathbf{q}_{\perp}} G_{\mathbf{q}_{\perp}} G_{\mathbf{q}_{\perp}+\mathbf{k}_{\perp}}\left(1-I_{\mathrm{ir}}(\mathbf{k})\right)^{-1} \text { with } \\
& I_{\mathrm{ir}}(\mathbf{k})=\frac{\Delta}{d} N_{c} \int_{\mathbf{q}_{\perp}} G_{\mathbf{q}_{\perp}} G_{\mathbf{q}_{\perp}+\mathbf{k}_{\perp}} \sum_{n \neq 0} \sum_{m}(-1)^{m n} V^{(0)}\left(m, n, q_{y, j}\right) \mathrm{e}^{\mathrm{i} d n k_{x}} .
\end{aligned}
$$

Here $G_{\mathbf{q}_{\perp}}=\left(\mu+\xi_{0}^{2} \mathbf{q}_{\perp}^{2}\right)^{-1}$ is the propagator in $p \gg 1$ of the pair-field fluctuation in LLL. Keeping the lowest order terms in $\mathrm{e}^{-p}$ in the $n, m$-summations of eq.(2.18), the glass susceptibility is given by

$$
\chi_{G}(\mathbf{k}) \propto\left(r_{G}+\frac{\xi_{0}^{2}}{6 \mu} \mathbf{k}_{\perp}^{2}+1-\cos \left(2 k_{x} d\right)\right)^{-1}
$$

where $r_{G}=\alpha-1$ with $\alpha^{-1}=\Delta N_{c} \exp (-2 p) /(2 \pi \mu)$. If we focus on the $p$-region in which, due to point disorder, a first order transition ${ }^{-1)}$ accompanied by both the positional and (ordinary) superconducting orderings does not occur at $T_{m} \simeq T_{s c}$ (see below) any longer, the renormalized mass $\mu$ must remain positive (noncritical) even in $T<T_{m}$ because, as reflected in the expression (2.4), the LLL superconducting fluctuation is two-dimensional in nature. Owing to eq.(2.14), the 
mass-renormalization can be performed in the same manner as in ref.22, and one easily finds

$$
\mu=\mu_{0}+2 \pi \varepsilon_{G}^{(2)}(T)\left(\sum_{m} \mathrm{e}^{-p m^{2}}\right)^{2} \int_{\mathbf{q}_{\perp}} \frac{1}{\mu+\mathbf{q}_{\perp}^{2}}\left(\beta_{A}-\Delta_{\text {eff }}\left(\beta_{A}-1\right)\right) .
$$

Since $\beta_{A}-1 \sim \mathrm{O}\left(\mathrm{e}^{-p}\right) \ll 1$ due to the vortex-confinement between neighboring layers, we can neglect the last term of eq.(2.20) even at low enough temperatures so that the solution becomes $\Delta$-independent. The resulting $\mu$ can be expressed below the mean field phase boundary in the form

$$
\mu \simeq \frac{\varepsilon_{G}^{(2)}(T)}{2} \exp \left(\frac{2 \tilde{\mu}_{0}}{\varepsilon_{G}^{(2)}(T)}\right)
$$

Here $\tilde{\mu}_{0}=\mu_{0}+\varepsilon_{G}^{(2)}(T) \ln \left(2 \mu_{c} / \varepsilon_{G}^{(2)}(T)\right) / 2$, and $\mu_{c}$ is a cutoff constant of $\mathrm{O}(1)$.

Using this $\mu$-expression, the uniform glass susceptibility $\chi_{G}(\mathbf{k}=0)$ is found to diverge at the Josephson-vortex-glass (JG) transition temperature $T_{J G}(B)$, where

$$
\frac{T_{c 2}-T_{J G}}{T_{J G}}=2 \pi \varepsilon_{G}^{(2)} \frac{d^{2} \sqrt{\Gamma}}{\phi_{0}}\left(B-B_{c}^{(\mathrm{in})}(\Delta)\right)
$$

where $\varepsilon_{G}^{(2)}=16 \pi^{2} \lambda^{2}(0) k_{\mathrm{B}} T_{c 0} /\left(\phi_{0}^{2} d\right)$ is the $2 \mathrm{D}$ fluctuation strength defined in $\S 1$, and $T_{c 2}$ is the mean field transition line. For simplicity, the correlation volume $N_{c}$ included in the characterstic field

$$
B_{c}^{(\mathrm{in})}(\Delta) \simeq \frac{\phi_{0}}{4 \pi d^{2} \sqrt{\Gamma}} \ln \left(\frac{\Delta N_{c}\left(\Delta_{\mathrm{eff}}\right)}{2 \pi \mu_{c}}\right),
$$

which is the only measure of a disorder strength in eq.(2.22), is assumed here not to be $B$-dependent (see, however, $\S 4$ ). In very clean systems, $B_{c}^{(i n)}$ becomes quite large, while it decreases with increasing disorder and may become negative in moderately disordered case because the inequality, $\varepsilon_{G}^{(2)}, \Delta<1$, is expected even in real systems with strong thermal fluctuation such as BSCCO. As clearly seen in the above discussion, eq.(2.22) is well defined in $B>B_{c}^{(\text {in) }}$ and in $T_{J G}<T_{m}$. The JG transition temperature $T_{J G}$ approaches the melting line $T_{m}$ as $B$ decreases and approaches $B_{c}^{(\text {in) }}$, or equivalently, as the disorder becomes weaker at a fixed $B$ value, i.e., when $N_{c}$ grows and approaches $N_{d}$. Oppositely, an increase of $B$ leads to a decrease of $N_{c}$, and hence, $T_{J G}(B)$ goes away from $T_{m}$. In high enough fields, $N_{c}(\Delta)$ reduces to a constant of order unity as in $\mathbf{B} \| \mathbf{c}$, and eq. (2.22) is simplified in the form

$$
\frac{\tilde{T}_{c 2}-T_{J G}(B)}{T_{J G}(B)}=\frac{\varepsilon_{G}^{(2)}}{1-\varepsilon_{G}^{(2)} \ln \sqrt{\Delta / 2 \pi}} \frac{2 \pi d^{2} \sqrt{\Gamma}}{\phi_{0}} B,
$$

where $\tilde{T}_{c 2}=T_{c 0} /\left(1-\varepsilon_{G}^{(2)} \ln \sqrt{\Delta / 2 \pi}\right)$. Note the weak dependence of $T_{J G}$ on the pinning strength $\Delta$.

It is valuable to point out that, except a difference in the numerical prefactor of order unity multiplying $B$, eq.(2.22) is of the same form as a $B-T$ line following from the disorder-free Lindemann criterion

$$
<s_{y}^{2}>_{\text {har }} \sim \frac{r_{B}^{4}}{d^{2}}
$$


if $B_{c}^{(\mathrm{in})}$ in eq. $(2.21)$ is replaced by $B_{\mathrm{el}}^{(\mathrm{in})} \simeq-\left(\phi_{0} /\left(2 \pi d^{2} \sqrt{\Gamma}\right)\right) \ln \left[\mathrm{e}^{4} /\left(\left|\mu_{0}\right| \pi^{4}\right)\right]$. Here, $s_{y}=r_{B}^{2} \partial_{x} \chi$ (see ref.26) is the displacement fluctuation parallel to the layers, and $\chi$ is the harmonic phase fluctuation around the $w=1$ regular solid, i.e., the disorder-free ground state in $p>1$, with its fluctuation energy

$$
\delta \mathcal{H}_{L D}^{(w=1)}=\frac{k_{\mathrm{B}} T_{c 0}\left|\tilde{\mu}_{0}\right|}{2 \pi \varepsilon_{G}^{(2)} d} \int \mathrm{d}^{3} r\left(\sum_{\mu=y, z}\left(\partial_{\mu} \chi\right)^{2}+\xi_{0}^{-2}\left|\tilde{\mu}_{0}\right| \mathrm{e}^{-p}\left(d^{2} \partial_{x}^{2} \chi\right)^{2}\right)
$$

(see eq.(3.8) of ref.5). The thermal average $<\ldots \ldots>_{\text {har }}$ in eq.(2.25) is defined in terms of this fluctuation energy. Further, we used in eq.(2.25) the fact that the average spacing between the vortices in $y$ direction of the $w=1$ solid is $2 \pi r_{B}^{2} / d$.

To simplify our discussion on the result (2.22), we assume for a moment $B_{c}^{(\mathrm{in})} \ll \phi_{0} /\left(2 \pi d^{2} \sqrt{\Gamma} \varepsilon_{G}^{(2)}\right)$ (i.e., $\left.\varepsilon_{G}^{(2)} \ln \sqrt{\Delta / 2 \pi} \ll 1\right)$ and hence, identify eq.(2.22) with

$$
T_{c 2}-T_{\mathrm{el}}(p) \simeq T_{\mathrm{el}}(p) \varepsilon_{G}^{(2)} p .
$$

This $T_{\mathrm{el}}(B)$ decreases and approaches zero with increasing $p$. On the other hand, the superconducting transition in each layer in the disorder-free case may occur at a nozero temperature even in large enough $p(\propto \sqrt{\Gamma})$ because the 2 D Meissner response is permitted 27 Thus, the disorder-free superconducting transition point $T_{\mathrm{sc}}$ should exist far above $T_{\mathrm{el}}$, at least in large enough $p$, and the only possible parameter-dependence determining such a $T_{\mathrm{sc}}$ will be

$$
T_{c 2}-T_{\mathrm{sc}} \simeq T_{\mathrm{sc}} \varepsilon_{G}^{(2)}
$$

Further, the solidification (melting) transition at $T_{m}$ was argued in ref.5, as in $\mathbf{B} \| \mathbf{c}$ case, to be of first order and simultaneously the superconducting transition, implying that $T_{m}=T_{\mathrm{sc}}$, as far as the thermally excited vortex loops are negligible (see Note added in poof in ref.5). Thus, if the first order freezing transition line at $T_{m}$ terminates, for instance, due to the point disorder, somewhere in the $B$ - $T$ phase diagram of a sample, the ordinary superconducting ordering in $\mathbf{B} \perp \mathbf{c}$ will not occur at any nonzero temperature in this sample.

However, a possibility (in the disorder-free case) of a splitting between the superconducting and melting transitions (i.e., $T_{m}<T_{\mathrm{sc}}$ ) was not excluded there, at least if going beyond the LLL approximation. If $T_{m}<T_{\mathrm{sc}}$, this melting transition, occuring between two superconducting phases, is associated only E $_{\text {) }}$ with the positional ordering across the layers, and $T_{m}$ will decrease with increasing $p$. A reasonable guess of such a $T_{m}$ will be that $T_{m} \propto T_{\mathrm{el}}(p)$. However, it was argued28) that a $B-T$ line obtained in terms of a Lindemann criterion neglecting vortex displacements across the layers should be far below a true melting transition in $\mathbf{B} \perp \mathbf{c}$. Actually, such a possibility that $T_{m} \simeq T_{\mathrm{el}}(p)<T_{\mathrm{sc}}$ in $p>1$ is unreasonable even from another point of view. To explain this, let us first point out that the expression (2.27) on $T_{\mathrm{el}}$ is independent of the starting model and valid in the phase-only approximation, as far as the interlayer shear modulus is small like29) $\sim \mathrm{e}^{-p}$, because the only model-dependence appears, in such a harmonic fluctuation energy, as a 
prefactor of the interlayer shear energy, while such a prefactor is reflected merely logarithmically in a quantity corresponding to $B_{\mathrm{el}}^{(\mathrm{in})}$ or $B_{c}^{(\mathrm{in})}$ and hence, is negligible for large enough $p$. On the other hand, the melting line $T_{m}^{(c)}(B)$ in $p \ll 1$, where the layering effect may be negligible, will be well approximated by the corresponding curve of the anisotropic GL mode 26 ) in $\mathbf{B} \perp \mathbf{c}$, which is, in the phase-only approximation, given by

$$
T_{c 2}-T_{m}^{(c)}(p) \simeq T_{m}^{(c)}(p) \varepsilon_{G}^{(2)} \sqrt{p}
$$

except a numerical prefactor of order unity of r.h.s. (see eq.(2.18) of ref.26). Thus, as shown in Fig.1, just three curves expressed by eqs.(2.27) to (2.29) are expected in the phase-only approximation of the LD model. Note that the point $p \sim 1$, at which the three curves merge with each other, is a kind of dimensional (3 D to $2 \mathrm{D}$ ) crossover of the melting line in $\mathbf{B} \perp \mathbf{c}$ and corresponds to the field 30 ) $B_{d c}^{\prime} \sim \phi_{0} / d^{2} \Gamma$ in $\mathbf{B} \| \mathbf{c}$ case. Such a crossover to the $2 \mathrm{D}$ regime in $\mathbf{B} \| \mathbf{c}$ is expected to occur with increasing $\Gamma$ in order to avoid a unlimited enhancement of thermal fluctuation (Note that

the $B=0$ fluctuation strength $\sqrt{\varepsilon_{G}^{(3)}} \propto \varepsilon_{G}^{(2)} \sqrt{\Gamma}$ in $3 \mathrm{D}$ grows unlimitedly as $\Gamma$ increases). Besides this, in the present $\mathbf{B} \perp \mathbf{c}$ case, we have not only such a quantitative field-induced enhancemnet of fluctuation but also a qualitative field-induced reduction of the fluctuation due to the (1 D to $2 \mathrm{D})$ rise $\mathrm{E}$ ) of its dimensionality. Thus, it is quite unreasonable to expect that $T_{m}$ in $\mathbf{B} \perp \mathbf{c}$ and $p \gg 1$ would be given by $T_{\mathrm{el}}(p)$ far below (the extrapolated) $T_{m}^{(c)}$.

Now, it will not be difficult any longer to understand why the vortex-glass transition position $T_{J G}(p)$ in $p \gg 1$ is related to the pinning-free Lindemann criterion (2.25). First, the small factor $\sim \mathrm{e}^{-p}$ is common both to the interlayer shear modulus in the elastic energy and the prefactor in $I_{\text {ir }}$ of the glass susceptibility (see eq.(2.18)), and its origin is a weak spatial variation of $|\psi|$ in $y$ direction, i.e., a (small but) positive $\beta_{A}-1$, in the solid state. The relation $\beta_{A}-1 \sim \mathrm{e}^{-p}$ may remain unchanged as far as the disorder is weak so that a granular structure, i.e., a spatial variation of $|\psi|$ induced by $\Delta$ at low enough $T$, on the layers is invisible. Further, the rise, due to the layering, of the dimensionality of superconducting fluctuation is also reflected in the dispersion of the elastic energy, and the resulting exponential $T$-dependence, combining with the above-mentioned exponential $p$ dependence, makes a role of disorder in the resulting transition line weak logarithmically. The only effect of the point disorder on the vortex elasticity near $T_{m}$ and above $T_{J G}$ (i.e., in a vortexslush regime 32 $)$ is regarded as a renormalization on the interlayer shear modulus $\propto \mathrm{e}^{-p}$. When combined with the model-independence of the $T_{\mathrm{el}}(p)$-line, this correspondence between $T_{J G}(p)$ and the elastic temperature $T_{\mathrm{el}}(p)$ convincingly suggests that the behavior $T_{c 2}-T_{J G}(B) \sim T_{J G}(B) \varepsilon_{G}^{(2)} p$ may be generally valid irrespective of our use of the LLL approximation.

\section{§3. Responses Quantities near $T_{J G}$}

In this section, we examine linear response properties near and above the transition point $T_{J G}$. As mentioned in relation to eq.(2.4), the physical quantities in the vortex liquid region with $\mathrm{e}^{-p} \ll 1$ 
are insensitive to the field $B$, reflecting the confinement of vortices in the spacings between two neighboring layers in $p \rightarrow \infty$, while in the vortex-slush region 1.32) above $T_{J G}$ but below $T_{m}$ they should depend on $B$ due to the remarkable $B$-dependence of $T_{J G}(B)$ given by eq.(2.22).

This picture on the $B$-dependence also applies to the in-plane resistivities $\rho_{\mu \mu}(\mu=y$ or $z)=$ $1 /\left(\sigma_{n}+\sigma_{s, \mu \mu}\right)$, where $\sigma_{n}$ is the normal part (quasiparticle contribution) of the in-plane conductivity and reasonably assumed to be $\mu$-independent (i.e., isotropic in $y$ - $z$ plane). Since, just as in the case with no disorder (see the discussion leading to eq.(2.6)), the gauge-coupling and the gradient terms in $\mathrm{e}^{-p} \ll 1$ is approximated in an isotropic form in $y$ - $z$ plane, and the $\mathbf{q}_{\perp}$ dependence of the bare four-point vertices in the replicated hamiltonian in such high fields will merely play secondary roles, the total conductivities in high fields $\mathbf{B} \perp \mathbf{c}$ are also isotropic in $y$ - $z$ plane. Namely, we have the "force free" behavior in $\mathrm{e}^{-p} \ll 1$ even when the point disorder is not negligible. Further, the recovery of the gauge-coupling form in $y$ - $z$ plane (see eq.(2.4)) within LLL also implies that this gauge-invariant gradient operates on the glass order parameter formed within LLL. This enables 1 ) us to, just like the conductivity (]) parallel to $\mathbf{B}$ in $\mathbf{B} \| \mathbf{c}$ case, anticipate the diverging behaviors of $\sigma_{s, \mu \mu}$ near $T_{J G}$ according to the scaling argument same as in the zero field transition, and consequently, we expect the critical behavior

$$
\sigma_{s, \mu \mu} \sim\left(T-T_{J G}(B)\right)^{\nu_{J}\left(1-z_{J}\right)}
$$

where $z_{J}(>4)$ and $\nu_{J}(>1 / 2)$ are, respectively, the dynamical exponent and the exponent of the correlation length, $\xi_{J}(T) \sim\left(T-T_{J G}(B)\right)^{-\nu_{J}}$, describing the JG critical behavior. In $p \gg 1$, the behavior (2.6) far above $T_{J G}$ (but below $T_{c 0}$ ) is expected to, on cooling, smoothly change close to $T_{J G}$ into the behavior (3.1) which is also isotropic in $y-z$ plane. Note, however, that, due to the $B$-dependence of $T_{J G}(B)$, the in-plane resistivity should show a $B$-dependent "force free" behavior near $T_{J G}$.

We will focus in the remainder of this section on the static responses to a tilt of the applied field which are measures of the presence or absence of a transverse Meissner effect (TME) in the resulting glass (JG) phase. This static tilt response is a key quantity for distinguishing $\mathbb{A}^{\text {b }}$ between various glass phases and is in this sense more nontrivial than the resistivities which vanish at a glass transition irrespective of the current direction. The static tilt response $\Delta C_{44, x}$, corresponding to the single vortex part of a tilt modulus, in the $x$-direction is defined 30 by

$$
\Delta C_{44, x}=\left.B^{2} \frac{\Upsilon_{y}\left(k_{x}=k_{y}=0, k_{z}\right)}{k_{z}^{2}}\right|_{k_{z}=0}
$$

where $\Upsilon_{y}\left(k_{x}=k_{y}=0, k_{z}\right)$ is the helicity modulus defined by a gauge disturbance $\delta A_{y}(z)$ dependent only on $z$, and the corresponding $\Delta C_{44, y}$ is similarly defined in terms of $\delta A_{x}(z)$. Another kind of 
tilt moduli are defined in terms of $\delta A_{z}$ in the form 3 )

$$
C_{44, x}^{\prime}=\left.B^{2} \frac{\Upsilon_{z}\left(k_{x}=0, k_{y}, k_{z}=0\right)}{k_{y}^{2}}\right|_{k_{y}=0}
$$

which is a response to $\delta A_{z}(y)$. In the disorder-free liquid regime in $\mathbf{B} \| \mathbf{c}, \Delta C_{44, \mu}$ coincides 30 with $C_{44, \mu}^{\prime}$, and $\Delta C_{44, x}$ in the (disorder-free) liquid regime in $p \gg 1$ is expected to, just as $\sigma_{s, \mu \mu}$ (see eq.(2.6)), grow exponentially on cooling, i.e.,

$$
\Delta C_{44, x} \sim \exp \left(T_{c 0}\left(\varepsilon_{G}^{(2)}\right)^{-1} / T\right) .
$$

First, we will anticipate a critical behavior of $\Delta C_{44, x}$ with no detailed calculation and simply by comparing with $C_{44, x}^{\prime}$. To do this, one only has to note that the derivation of the quadratic terms of eq.(2.4), in which $\delta A_{\mu}(\mu=y$ and $z)$ are constant, is trivially extended to the case with a $z$-dependent $\delta A_{y}$ and a $y$-dependent $\delta A_{z} \sim \delta a\left(q_{y}\right) \mathrm{e}^{\mathrm{i} q_{y} y}$ with $\left|q_{y}\right| \ll d / r_{B}^{2}$. Namely, in this case the quadratic term of eq.(2.4) is replaced with

$$
\begin{gathered}
\tilde{\mathcal{H}}_{\mathrm{LD}, 2}(\varphi)=\int \mathrm{d} y \mathrm{~d} z \sum_{n}\left(\xi_{0}^{2}\left|\left(-\mathrm{i} \partial_{y}-\frac{2 \pi}{\phi_{0}} \delta A_{y}(z)\right) \varphi\left(n, \mathbf{r}_{\perp}\right)\right|^{2}+\mu_{0}\left|\varphi\left(n, \mathbf{r}_{\perp}\right)\right|^{2}\right. \\
\left.+\xi_{0}^{2}\left|\left(-\mathrm{i} \partial_{z}-\frac{2 \pi}{\phi_{0}} \delta A_{z}(y)\right) \varphi\left(n, \mathbf{r}_{\perp}\right)\right|^{2}\right)
\end{gathered}
$$

where the real space representation for $\varphi$-fields is defined here by assuming any $y$-dependence to have wavelengths longer than $r_{B}^{2} / d$. As explained elsewhere, A $^{2}$ when a vortex-glass transition will occur within LLL, the presence of a gauge-invariant gradient $-\mathrm{i} \partial_{\mu}-\left(2 \pi / \phi_{0}\right) \delta A_{\mu}$ operating on the LLL pair-field in a hamiltonian such as eq.(2.4) or (3.5) implies that the corresponding gauge-invariant gradient operating on the glass order parameter must appear in a resulting effective hamiltonian $\mathcal{H}_{\text {eff, } G}$ expressed only by the glass order parameter, because the analysis in LLL leading to $\mathcal{H}_{\text {eff, } G}$ is formally the same as the corresponding one in zero field case. Since this similarity to the zero field normal-Meissner transition implies that the scaling argument 16 on the critical behavior of the conductivity $\sigma_{s, \mu \mu}$ near the glass transition is applicable just as in zero field case, we have concluded $\Phi^{-1}$ that the helicity modulus $\Upsilon_{z}(\mathbf{q}=0)$ parallel to $\mathbf{B}$ should be finite in any vortex-glass phase. Since, in the present case, this applies also to the $y$-direction due to the recovery of the isotropy in $y-z$ plane, we can conclude that, as in any pinned Josephson vortex solid with no disorder, 而 $\Upsilon_{y}(\mathbf{q}=0)$ should be also finite in the JG phase. Based on eq.(3.2), this directly implies that the JG phase should have a TME perpendicular to the layers, and consistently that the tilt response (i.e., a diamagnetic susceptibility 30) $\Delta C_{44, x}$ should diverge, just as in the normal-Meissner transition, in proportional to the correlation length $\xi_{J G}(T)$ on approaching $T_{J G}$ from above,

$$
\Delta C_{44, x} \propto \xi_{J G}(T) \sim\left(T-T_{J G}(B)\right)^{-\nu_{J}}
$$


which will reduce to eq.(3.4) far above $T_{J G}(B)$ in $p \gg 1$.

Next, let us turn to $\Delta C_{44, y}$. In this case, it is convenient to return to the original model (1.2) with no $\delta A_{\mu}(\mu=y, z)$ and with replacement $\psi_{j} \rightarrow \psi_{j} \exp \left(\mathrm{i} d 2 \pi j \delta A_{x}(z) / \phi_{0}\right)$. After gauge-transforming $\psi(x) \exp \left(\mathrm{i} 2 \pi x \delta A_{x}(z) / \phi_{0}\right) \rightarrow \psi(x)$ in the second representation of eq.(1.2) and, for simplicity, assuming $\delta B_{y}=\partial_{z} \delta A_{x}$ to be constant, we have

$$
\begin{gathered}
\mathcal{H}_{L D}=\int \mathrm{d}^{3} r \sum_{m=-\infty}^{+\infty} \mathrm{e}^{\mathrm{i} 2 \pi m x / d}\left[\varepsilon_{0}|\psi(\mathbf{r})|^{2}+\xi_{0}^{2} \sum_{\mu=y, z}\left|\left(-\mathrm{i} \partial_{\mu}+\frac{2 \pi}{\phi_{0}}\left(B \hat{y}-\delta B_{y} \hat{z}\right) x\right) \psi(\mathbf{r})\right|^{2}\right. \\
\left.+\Gamma^{-1}\left(\frac{\xi_{0}}{d}\right)^{2}|\psi(\mathbf{r})-\psi(\mathbf{r}+d \hat{x})|^{2}+\frac{b}{2}|\psi|^{4}\right]
\end{gathered}
$$

in place of the second representation of eq.(1.2). When the disorder term (1.3) is included in addition to eq.(3.7), the inclusion of $\delta B_{y}$ merely implies a rotation of the applied field direction within $y$ - $z$ plane in which the original system in zero field is isotropic. Namely, since this disturbance is the same as a tilt in an isotropic $3 \mathrm{D}$ system with point disorder, no TME is expected for a tilt within the layers, i.e., $\Delta C_{44, y}$ is nondivergent at and below $T_{J G}$. Note that this conclusion was obtained without using the LLL approximation and hence, should be valid at any $p$-value.

In contrast to this nondivergent $\Delta C_{44, y}$, the above conclusion on the divergent $\Delta C_{44, x}$ was obtained in terms of the high $p$ approximation. Hence, one may suspect that a vortex-glass phase in lower $p$-values, where the layering is quantitatively negligible at least above $T_{m}$, will have no TME as well as the ordinary vortex-glass (VG) phase.16) However, as mentioned below eq.(2.2), the cross term $\sim-\varphi^{*} \mathrm{i} \delta A_{y} \partial_{y} \varphi$, which determines the current vertices in the response function, exists within LLL even without assuming a large $p$. This rather suggests the presence, irrespective of the $p$-value, of TME for a tilt perpendicular to the layers in the ordered phases.

To clarify these points, we examine the tilt responses in the uniaxially periodic GL model 18 with point disorder

$$
\mathcal{H}_{\mathrm{per}}=\mathcal{H}_{\mathrm{GL}}-\int \mathrm{d}^{3} r\left[u_{p} \cos (2 \pi x / d)-u_{r}(\mathbf{r})\right]|\psi(\mathbf{r})|^{2},
$$

where $\mathcal{H}_{\mathrm{GL}}$ is the ordinary isotropic GL model, the positive constant $u_{p}$ is the strength of a periodic variation of $T_{c 0}$ playing similar roles to the layer structure of the LD model, and $u_{r}$ is a random potential expressing the point disorder and has the ensemble defined by $\bar{u}_{r}=0$ and $\overline{u_{r}\left(\mathbf{r}_{1}\right) u_{r}\left(\mathbf{r}_{2}\right)}=$ $\xi_{0}^{3} \Delta \delta^{(3)}\left(\mathbf{r}_{1}-\mathbf{r}_{2}\right)$. Hereafter, we assume a weaker magnetic field satisfying $r_{B} \geq d$ so that the ground state in a fixed field becomes a $w \geq 1$ pinned solid or the floating solid.0.33) Note that, in this model, the pair-field can be nonzero not only on the "layers" but also between two neighboring "layers" and hence that the point disorder existing between the layers is also effective as pinning sites enabling the vortex lines to locally deviate from directions parallel to the layers. By contrast, in the LD model the point disorder, as well as the pair-field, is restricted on the layers and hence, will not play any role for releasing the vortices locked in the layers. Due to this difference, described 
in Fig.2, in the disorder configurations between the two models, one may expect that the point defects existing between the neighboring "layers" in the periodic GL model compete with the layer structure inducing司) the nonvanishing helicity modulus $\Upsilon_{y}(\mathbf{q}=0)$, implying TME perpendicular to the layers, and that this TME perpendicular to the layers may be destroyed by such defects absent in the LD model. Namely, a TME should occur more easily in the LD model than in the periodic GL model. Keeping this in mind, let us examine $\Delta C_{44, \mu}$ of the model (3.8) in a manner similar to in ref.4.

As in previous works 1 (4) on glass transitions in $\mathbf{B} \| \mathbf{c}$, we will use a high field approximation convenient in examining electro-magnetic responses. When treating $\mathcal{H}_{\mathrm{GL}}$ by expressing $\psi$ in terms of the Landau levels (LLs), the vertex on an external current perpendicular to $\mathbf{B}$ is accompanied by the next lowest LL (NLL) even if the thermodynamics is described primarily by the LLL modes. Since this NLL mode is not associated with any ordering in vortex systems, 130 it remains massive and heavy in high fields in the sense that its time scale becomes much shorter than those of LLL modes. In the ideal case with no source of vortex pinnings, this short time scale of NLL inevitably becomes the time scale for all terms of the perturbation series of a conductivity perpendicular to $\mathbf{B}$, and consequently the vortex flow conductivity follows 34 This fact is clearly seen (1) particularly in the high field approximation where the LLL modes are assumed not to interact, through the $|\psi|^{4}$ term of $\mathcal{H}_{\mathrm{GL}}$, with higher LL modes. However, when either a periodic or a random pinning term is present as in eq.(3.8), a much longer time scale than that of NLL can be picked up from a section, composed only of the LLL modes, in Feynman diagrams contributing to a response quantity, and hence the presence of the time scale of NLL can be neglected in such diagrams. In refs.1 and 4, such diagrams with a long time scale growing on cooling due to a LLL vertex correction expressing the VG fluctuation were found for a conductivity perpendicular to $\mathbf{B}$ in the case with no periodic pinning effect. Its examples are given in Fig.3 (a), where the open square denotes the abovementioned LLL vertex correction corresponding to the glass susceptibility. Situation is similar in static responses such as $\Delta C_{44, \mu}$, and, just like the neglect of dynamics of the NLL modes in such diagrams of the conductivities, the $z$-dependence of the NLL fluctuation propagator can also be disregarded in examining $\Delta C_{44, \mu}$.

Once the periodic potential $\propto u_{p}$ is included, the linear responses become anisotropic in $x-y$ plane. When the gauge disturbance $\delta \mathbf{A}$ couples to the pair-field through the GL hamiltonian, the tilt response $\Delta C_{44, x}$ is generally expressed in the form 1, (4)

$$
\begin{aligned}
\Delta C_{44, x}=\frac{2 k_{\mathrm{B}} T}{V r_{B}^{2}}\left(-\frac{\partial}{\partial k_{z}^{2}}\right) \sum_{K, K^{\prime}}\left[\overline{<\varphi_{1, K+k_{z}} \varphi_{1, K^{\prime}+k_{z}}^{*} \varphi_{0, K^{\prime}} \varphi_{0, K}^{*}>}\right. \\
+\overline{\left.<\varphi_{1, K+k_{z}} \varphi_{0, K^{\prime}+k_{z}}^{*} \varphi_{1, K^{\prime}} \varphi_{0, K}^{*}>\right]},
\end{aligned}
$$

where $V$ is the system volume. The last (second) term of eq.(3.9) vanishes in the case isotropic in $x-y$ plane. For the present purpose, we need not to examine eq.(3.9) in details but only to 
focus on how the NLL fluctuation couples to a LLL fluctuation, in terms of the potential terms in (3.8), outside the LLL vertex correction implying the glass susceptibility in Feynman diagrams (see Fig.3). Let us consider the diagrams in Fig.3 (b), where the NLL lines couple to LLL lines only through the periodic potential, by expressing the pair-field in terms of the same representation of LLs as in the LD model (see the sentences prior to eq.(2.1)). The NLL-LLL coupling term, marked with dashed line in Fig.3, is written as

$$
-\sqrt{2} u_{p} \frac{2 \pi r_{B}}{d} \exp \left(-\left(\pi r_{B} / d\right)^{2}\right) \sum_{Q, q_{z}} \sin \left(\frac{2 \pi r_{B}^{2}}{d} q_{y}\right)\left(\varphi_{1}^{*}\left(n, \mathbf{q}_{\perp}\right) \varphi\left(n, \mathbf{q}_{\perp}\right)+\text { c. c. }\right),
$$

where $\varphi_{1}$ is the corresponding one in NLL to $\varphi$ in LLL. As already stated, the NLL fluctuation in Fig.3 (b) can be assumed to be independent of $z$. Then, when ensemble-averaging over the NLL modes according to Fig.3 (c), the resulting NLL propagator can be treated as a constant factor multiplying the current vertex, and the resulting $\mathrm{O}\left(\delta A_{y}\right)$ term $\left(\propto-u_{p} \delta A_{y} \sin \left(2 \pi r_{B}^{2} q_{y} / d\right)\left|\varphi\left(n, q_{y}, z\right)\right|^{2}\right)$ can be regarded as a current vertex defined within LLL. Explicitly, if using the fact that the NLL propagator $G_{1}\left(q_{y}, q_{z}=0\right)=<\left|\varphi_{1}\left(n, q_{y}, q_{z}=0\right)\right|^{2}>$ in high enough fields can be replaced,34 except corrections in $q_{y}^{2}$, by $r_{B}^{2} /\left(2 \xi_{0}^{2}\right)$ deep in the liquid regime, 34 the obtained dispersion of the LLL fluctuation and the current vertex coupled to $\delta A_{y}$ can be seen as those defined from the following quadratic term of an effective hamiltonian only on the LLL modes

$$
\begin{gathered}
\tilde{\mathcal{H}}_{\text {per }, 2}=\int \mathrm{d} y \mathrm{~d} z \sum_{n} \varphi^{*}\left(n, \mathbf{r}_{\perp}\right)\left(u_{p} \exp \left(-\left(\pi r_{B} / d\right)^{2}\right)\left(1-\cos \left[\frac{2 \pi r_{B}^{2}}{d}\left(-\mathrm{i} \partial_{y}-\frac{2 \pi}{\phi_{0}} \delta A_{y}(z)\right)\right]\right)\right. \\
\left.+\mu_{0}+\xi_{0}^{2}\left(-\mathrm{i} \partial_{z}-\frac{2 \pi}{\phi_{0}} \delta A_{z}(y)\right)^{2}\right) \varphi\left(n, \mathbf{r}_{\perp}\right) .
\end{gathered}
$$

Now, let us consider the diagrams in Fig.3 (c) composed of the current vertex of Fig.3 (b). In Fig.3 (c), the left (right) diagram arises from the first (second) term of eq.(3.9). Further, note that, near $T_{J G}$, the gauge invariant gradient term in $y$ direction has only to be kept up to quadratic order. Then, the argument given below eq.(3.3) can directly be used in the present case. The only difference from the LD case is the absence, at the quantitative level, of isotropy in $y$ - $z$ plane due to the difference in coefficients of the gradient terms. Since the strength $u_{p}$ is independent of other material parameters, $\Delta C_{44, x}$ in the model (3.7) is also predicted to diverge in proportional to a correlation length near the resulting glass transition. Contrary to this, this divergence is absent in $\Delta C_{44, y}$, because this quantity is given by replacing the plus sign prior to the last term in eq.(3.9) by a minus sign, and hence, the contributions corresponding to the two terms of Fig.3 (c) cancel with each other. We note that the diagrams, such as Fig.3 (d), including just one $u_{p}$-vertex coupling between the LLL and NLL modes do not give nonvanishing contributions because of the rule34) illustrated in Fig.3 (e). Hence, only the sum of diagrams such as Fig.3 (a) give a nonvanishing contributions related to the glass fluctuation which is easily verified to be nondivergent at the 
transition for the same reason as in the VG case 1 In passing, we note that what is essential to obtaining the above results is the presence of a nonzero gauge coupling $\sim \mathrm{i} \delta A_{y}(z) \varphi^{*} \partial_{y} \varphi$ after averaging over the NLL modes but is not the use of the approximation on $G_{1}$ in getting eq.(3.11).

Thus, we have shown that the divergent $\Delta C_{44, x}$, as well as the nondivergent $\Delta C_{44, y}$, is also realized in this periodic GL model. We emphasize that this conclusion is independent of the strength of the point disorder and of the strength $B$ of the magnetic field which tunes the structure of the disorder-free vortex solid in $\mathbf{B} \perp \mathbf{c}$ through a commesurability condition $\mathbf{0}, 2 \mathrm{2}, \mathrm{B}$ ) with the layer structure. Namely, the glass phase in the model (3.7) should have the anisotropic TME irrespective of $B$. Following the consideration based on Fig.2, this in turn convincingly suggests that our earlier prediction on the LD model that the resulting JG phase has TME only for tilts perpendicular to the layers is also likely to be valid for any $B$. Namely, the ground state will become the JG phase with a TME even in $p(<1)$-values where the disorder-free ground state may be a unpinned (floating) vortex solid, as far as the positional order in the resulting JG phase at such $p$-values is short-ranged. Further, we note that the presence of the TME perpendicular to the layers below $T_{J G}$ for any $p$ implies the presence in any $B$ of the so-called lock-in transition due to a tilting of the applied field in real systems with point disorder. Note that the so-called lock-in transition close to $\mathbf{B} \perp \mathbf{c}$ was discussed so far just in the pinning-free case. Since the floating solid possible in the disorder-free case has no TME, the above result implies that the point disorder destroying the positional order of this solid simultaneously creates the anisotropic TME in the real JG phase.

\section{$\S 4 . \quad \mathrm{B} \perp \mathrm{c}$ Phase diagram and Related Issues}

The resulting $\mathbf{B} \perp \mathbf{c}$ phase diagrams in this paper are sketched in Fig.4. The main point in our results is that the resulting $T_{J G}$ must decrease with increasing $B$ even in high fields satisfying $B>\phi_{0} / \sqrt{\Gamma} d^{2}$, while the first order melting temperature $T_{m}$ and phenomena in the vortex liquid regime above $T_{m}(B)$ in such high fields will be almost independent 5 ) of $p \propto B \sqrt{\Gamma}$, reflecting the nearly complete confinement of vortices within the interlayer spacings. The origin of the field dependence of $T_{J G}$ is a spatial variation of $|\psi|$ on the superconducting layers, which is small but nonvanishing in $B>\phi_{0} /\left(\sqrt{\Gamma} d^{2}\right)$, and, as already mentioned, is indispensable to a glass ordering. Since this spatial variation strongly depends on $p$ and disappears in high $B$ (or high $\Gamma$ ) limit of the LD model, $T_{J G}$ at which the resistivities vanish must depend on both $B$ and $\Gamma$ and approach zero in this limit. Namely, in $\mathbf{B} \perp \mathbf{c}$ case, $T_{J G}$ rapidly starts to deviate from the (disorder-free) melting line $T_{m}(B)$ with increasing $B$ even in clean systems, and hence, the resistive curves just above $T_{J G}$ but below $T_{m}$ also become $B$-dependent. This is quite different from the (ordinary) VG transition linet, (16) $T_{V G}(B)$ in $\mathbf{B} \| \mathbf{c}$ case, in which the VG line $T_{V G}(B)$ in higher fields (described as the lower dashed curves in Fig.7 of ref.1) yields the LLL scaling 34 ) similar to that of $T_{m}(B)$, and for this reason, this $T_{V G}(B)$ line is often regarded, for a practical purpose, as an extrapolation

of the $T_{m}(B)$ line by neglecting a narrow window 1 ) of the slush regime observed 24 recently in 
YBCO. Therefore, particularly in materials with stronger fluctuation, there may be a much wider vortex slush 132 regime in $\mathbf{B} \perp \mathbf{c}$ than in $\mathbf{B} \| \mathbf{c}$. Further, as shown in $\S 3$, the "force free" behavior itself in such high fields is never affected by the point disorder isotropic on the superconducting layers. Consequently, the in-plane resistivity curves in $p>1$ will show, near $T_{m}$, a crossover from a $B$-independent "force free" behavior to a $B$-dependent "force free" behavior below $T_{m}$. The $B$-dependent "force-free" resistive behavior below $T_{m}$ is qualitatively consistent with the behavior at lower temperatures seen in the data of ref.11.

For convenience, we have distinguished a dirtier case Fig.4 (a) from a cleaner case Fig.4 (b) based on whether a critical point of the first order transition line, denoted as $p=p_{c}$, satisfies $p_{c}<1$ or $p_{c}>1$. First, let us consider Fig.4(a). In this case, with increasing $p, N_{c}$ may already become of order unity before entering the $p>1$ region (Note that the shortest value of the positional correlation length perpendicular to the layers is the layer spacing $d$ and hence that one can say $\left.\left.N_{c}\right|_{\min } \sim \mathrm{O}(1)\right)$. Then, the relation $B_{c}^{(\mathrm{in})} \ll \phi_{0} /\left(2 \pi d^{2} \sqrt{\Gamma} \varepsilon_{G}^{(2)}\right)$ will be satisfied over a wide range in $p>1$, and we will have the relation $T_{c 2}-T_{J G} \propto T_{J G} B$ (see eq.(2.24)). Next, in turn, let us assume a cleaner case with $p_{c}>1$ such as Fig.4 (b). In this case, $N_{c}$ in $p<p_{c}$ will be of order $N_{d}$. As already mentioned, this means that the system below $p_{c}$ is in clean limit and that $T_{J G} \simeq T_{m}$ there (see Fig.4(b)). Next, when $p>p_{c}, N_{c}$ will rapidly decrease with increasing $p$, because the correlation volume will decrease with decreasing the shear modulus 290 ) $\sim \mathrm{e}^{-p}$ in the $w=1$ solid-like state. If naively assuming $N_{c} \sim \mathrm{e}^{-c_{1} p}$ with a constant $c_{1}>0$, the neglect in eq.(2.22) of a $B$-dependence of $B_{c}^{(\text {in) }}$ is not valid any longer. Only in $p$ values larger than $p_{c 1}, N_{c}$ will start saturating into a constant of order unity, and hence $B_{c}^{(\text {in) }}$ approaches a small positive or a negative constant. Namely, eq.(2.24) becomes valid only when $p>p_{c 1}$. Consequently, we have conjectured the phase transition curves, the solid curves in Fig.4 (a) and (b). It should be emphasized that the slope of the behavior $B_{J G} \sim T_{c 0}-T$ to be seen below $T_{m}$ is gentler as the fluctuation is stronger and is insensitive to the pinning strength. This will become useful for examining $T_{J G}(B)$ experimentally and implies that the vortex slush region (1, 32) to be seen experimentally becomes wider in materials with stronger fluctuation. According to ref.1, such an extension of the temperature window of the vortex slush region due to an enhanced fluctuation should also occur in $\mathbf{B} \| \mathbf{c}$ case, (1) contrary to the Bragg glass scenario 35 of the $\mathbf{B} \| \mathbf{c}$ phase diagram, and, in fact, was recently observed 24 through a doping dependence of the $B-T$ phase diagram of YBCO.

Theoretically, studies of a glass phase in $\mathbf{B} \perp \mathbf{c}$ were performed in previous works, 且) by assuming a dislocation-free glass phase. However, it was argued 36) that the resulting transition is not accompanied by a divergence of a glass correlation length, just like a vortex liquid-Bragg glass transition argued as a scenario of the first order transition in $\mathbf{B} \| \mathbf{c}$ case. Further, since the assumption in refs. 2 and 3 that the vortices cannot move across the layers is valid only in $\mathrm{E} p>1$, the situation

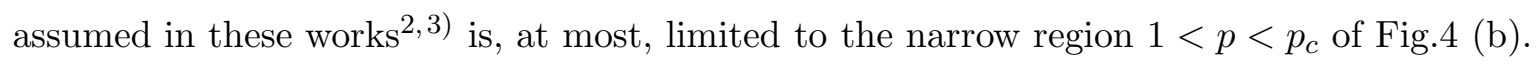


As shown in ref.5, the positional correlation of vortices perpendicular to $\mathbf{B}$ in $\mathbf{B} \perp \mathbf{c}$ first develops, on cooling, in $y$ direction parallel to the layers. This is a consequence of the recovery of isotropy in $y-z$ plane in high enough fields, emphasized in $\S 2$, which is essential to understanding the force-free behavior11 122 of the in-plane resistivities. On the other hand, the above-mentioned feature that the positional ordering first grows not across but rather along the layers contradicts the conjecture 37 ) favoring a smectic liquid phase. Namely, the smectic liquid picture 37) is incompatible with the force free resistive behavior.

In ref.38, $\mathrm{Hu}$ and Tachiki still argue that the disorder-free superconducting transition in $\mathbf{B} \perp \mathbf{c}$ is continuous for cases with larger anisotropy in which the anisotropy-induced vortex loop excitations seem to play an important role in the transition, while it is reasonably of first order for smaller anisotropies (see Note added in proof of ref.5). It is remarkable that the transition temperature they found is insensitive, at a fixed field, to the anisotropy even in $1.5<p<2$ studied in ref.38 (see Fig.3 there-in). This is consistent with our prediction $\mathbf{E}^{\mathrm{E}}$ that, in $p>1, T_{m}(p)$ approaches $T_{s c}$ independent of $p \propto B \sqrt{\Gamma}$, because, as commonly seen in various work14,28, 29) on the phaseonly model, the magnetic field $B$ always appears only as the parameter $p$. Hence, the transition temperature in ref.38 is identified with $T_{m} \simeq T_{s c}$. Clearly, this transition temperature has nothing to do with the observed 19.39) superconducting transition temperature in BSCCO which significantly decreases with increasing $B$ and also depends 19 much on the doping level, i.e., on the anisotropy (see below).

After preparing the first draft of the present paper, we were aware of recent data of a.c. susceptibility 19 and the resistivities, 39, (40) both of which can be direct probes of a glass transition, in field configurations including $\mathbf{B} \perp \mathbf{c}$ case of clean crystals of high $T_{c}$ superconductors. We will not discuss here complicated behaviors39) in fields tilted from the layers but focus on the data in $\mathbf{B} \perp \mathbf{c}$ relevant to the present work. In ref.19, the superconducting transition field in $\mathbf{B} \perp \mathbf{c}$ was determined in BSCCO crystals as the position at which the lock-in behavior (i.e., the TME in $\mathbf{B} \perp \mathbf{c}$ studied in $\S 3)$ seen in a.c. susceptibility data disappears with increasing the in-plane field.

The resulting line $H_{\|}^{c r}(T)$ roughly obeys $\Gamma H_{\|}^{c r} \sim\left(T_{c 0}-T\right) / T$, which is the same one as eq. (2.24) or (2.27) if the prefactor $\Gamma$ is replaced by $\sqrt{\Gamma} \varepsilon_{G}^{(2)}$. However, this difference in the prefactor does not preclude this identification between $H_{\|}^{c r}$ and $B_{J G}$ because the fluctuation strength $\varepsilon_{G}^{(2)}$ proportional to the squared penetration depth also increases, as well as the anisotropy $\Gamma$, with decreasing the doping level. Using a resonable $\varepsilon_{G}^{(2)}$ value in under-doped BSCCO, $\varepsilon_{G}^{(2)} \simeq 0.2$, corresponding to the in-plane penetration depth $\lambda(T=0) \sim 3000(A)$, the $H_{\|}^{c r}(T)$-line in the underdoped case of ref.19 is quantitatively consistent with eq.(2.27), i.e., $B_{J G}(T)$ obtained by assuming $B_{c}^{(i n)} \ll$ $\phi_{0} /\left(2 \pi d^{2} \sqrt{\Gamma} \varepsilon_{G}^{(2)}\right) \simeq 3(\mathrm{~T})$. On the other hand, the corresponding transition fields in BSCCO were determined in ref.39 as the positions at which the in-plane resistance at $H_{c}=0$ vanishes. According to ref.39, this transition is continuous. The resulting transition field in an optimally- 
doped sample seems to be comparable with the data in ref.19 and is roughly linear in temperature just like eq.(2.27). Further, in resistive measurements in YBCO,40 resistivities in all directions were found to vanish at the same temperature $T_{i}$. This is an evidence of a continuous JG transition occuring below the disorder-free melting line (see $\S 1$ ). It seems to us that, in ref.40, the outof-plane resistivity $\left(\rho_{c}\right)$ vanishes more rapidly (with a smaller exponent) compared with the inplane resistivities. Explaining this behavior seems to be theoretically interesting and will be tried elsewhere.

\section{Acknowledgement}

We gratefully acknowledge an informative discussion with T. Ishiguro and S. Nakaharai. This work was supported by a grant for CREST from Japan Science and Technology Corporation.

[1] R. Ikeda, J. Phys. Soc. Jpn. 65 (1996) 3998.

[2] D. Carpentier, P. Le Doussal, and T. Giamarchi, Europhys. Lett. 35 (1996) 379.

[3] J. Kierfeld, T. Nattermann, and T. Hwa, Phys. Rev. B 55 (1997) 626.

[4] R. Ikeda, J. Phys. Soc. Jpn. 69 (2000) 559.

[5] R. Ikeda and K. Isotani, J. Phys. Soc. Jpn. 68 (1999) 599.

[6] W. K. Kwok et al., Phys. Rev. Lett. 72 (1994) 1088.

[7] M. Charalambous et al., Phys. Rev. B 45 (1992) 5091.

[8] T. Ishida et al., Phys. Rev. B 58 (1998) 5222.

[9] N. E. Hussey et al., Phys. Rev. B 59 (1999) R11668.

[10] Y. Iye, S. Nakamura, and T. Tamegai, Physica C 159 (1989) 433.

[11] K. Kadowaki, Physica C 185-189 (1991) 1811.

[12] Y. Iye, T. Tamegai, and S. Nakamura, Physica C 174 (1991) 227.

[13] P. Kes, J. Aarts, V.M. Vinokur, and C.J. van der Beek, Phys. Rev. Lett. 64 (1990) 1063.

[14] G. Blatter, B. I. Ivlev, and J. Rhyner, Phys. Rev. Lett. 66 (1991) 2392.

[15] R. Ikeda, Physica C 201 (1992) 386.

[16] D.S. Fisher, M.P.A. Fisher, and D.A. Huse, Phys. Rev. B 43 (1991) 130.

[17] R. Ikeda, J. Phys. Soc. Jpn. 68 (1999) 729.

[18] A.M. Thompson and M. A. Moore, Phys. Rev. B 57 (1998) 13854.

[19] S. Nakaharai, T. Ishiguro, S. Watauchi, J. Shimoyama, and K. Kishio, Phys. Rev. B 61 (2000) 3270.

[20] R.A. Klemm, A. Luther, and M.R. Beasley, Phys. Rev. B 12 (1975) 877.

[21] For instance, T. C. Lubensky, in ill-condensed matter, ed. by R. Balian et al. (North-Holland, 1979 ), page 433.

[22] R. Ikeda, J. Phys. Soc. Jpn. 65 (1996) 1170.

[23] A.T. Dorsey, M. Huang, and M.P.A. Fisher, Phys. Rev. B 45 (1992) 523.

[24] T. Nishizaki et al., J. Low Temp. Phys. 117 (1999) 1375 and private communication.

[25] Further, note also that the quantity $\beta_{A}-1$ relevant to the positional ordering becomes significantly smaller with increasing $p(>1)$ due to the layering (or, an effect of the uniaxial periodic potential). It suggests that the jump of physical quantities at the solidification transition is weakened with increasing $p$. At a fixed magnitude of $\Delta_{\text {eff }}$, a weaker first order transition will be more easily destroyed by this pinning disorder. 
[26] R. Ikeda, Phys. Rev. B 46 (1992) 14842.

[27] J.M. Kosterlitz and D.J. Thouless, J. Phys. C 6 (1973) 1181; V. L. Berezinsky, JETP 34 (1972) 610.

[28] S. E. Korshunov and A. I. Larkin, Phys. Rev. B 46 (1992) 6395.

[29] B.I. Ivlev, N.B. Kopnin, and V.L. Pokrovsky, J. Low Temp. Phys. 80 (1990) 187.

[30] R. Ikeda, J. Phys, Soc. Jpn. 64 (1995) 1683.

[31] For simplicity, it was assumed in Fig.1 and 4 that the field $B$ appears everywhere in a phase diagram in the combination $p=2 \pi d^{2} \sqrt{\Gamma} B / \phi_{0}$. In particular, in systems with weaker fluctuation satisfying $\varepsilon_{G}^{(2)}<\Gamma^{-1}\left(\xi_{0} / d\right)^{2}$, the $3 \mathrm{D}$ LLL melting line $T_{m, \mathrm{~L}}(B)$ given by the ( $\Gamma$-independent) expression $T_{c 0}-T_{m, \mathrm{~L}} \sim\left(\varepsilon_{G}^{(2)} 2 \pi \xi_{0} d B / \phi_{0}\right)^{2 / 3}$ will interpolate between the low field line eq.(2.27) and the high field line (2.26). In contrast to B $\|$ c case, however, this LLL-scaling region seems to be quite narrow even in YBCO, and we will not try here a complicated description with this LLL-scaling region included.

[32] T.K. Worthington et al., Phys. Rev. B 46 (1992) 11854.

[33] The situation with $r_{B} \ll d$ is an appropriate model to quite different vortex states, for instace, of clean YBCO with parallel and periodic twin boundaries in B || c [W.K. Kwok et al., Phys. Rev. Lett. 76 (1996) 4596]. Our study for this case will be reported elsewhere.

[34] R. Ikeda, T. Ohmi, and T. Tsuneto, J. Phys. Soc. Jpn. 59 (1990) 1397; ibid. 60 (1991) 1051.

[35] T. Giamarchi and P. Le Doussal, Phys. Rev. B 55 (1997) 6577.

[36] T. Nattermann and S. Scheidl, to appear in Adv. Phys.. There, only the case with point disorder and fully magnetic screening was considered. According to the absence, suggested there, of a divergent glass correlation length in Bragg glass phases, the elastic approach 1 seems to be inapplicable to the cases with continuous glass transition.

[37] L. Balents and D.R. Nelson, Phys. Rev. B 52 (1995) 12951.

[38] X. Hu and T. Tachiki, cond-mat/0003068.

[39] J. Mirkovic, S.E. Savel'ev, E. Sugahara, and K. Kadowaki, preprint.

[40] S.A. Grigera et al., Phys. Rev. B 59 (1996) 11201. 


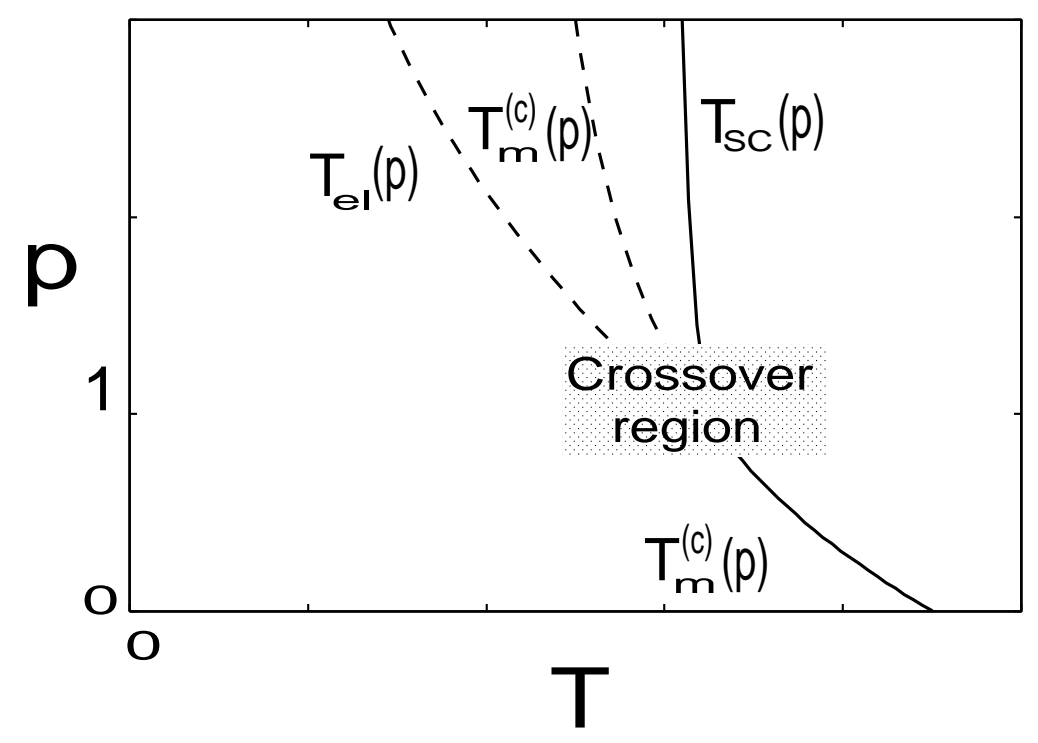

Fig. 1. Three possible characteristic curves, $T_{e l}(p), T_{m}^{(c)}(p)$, and $T_{s c}$, in the $p$ v.s. $T$ diagram of the phase-only approximation of the LD model. These curves merge roughly when $p \simeq 1$. The solid curve is believed to be the true transition curve in the disorder-free case.

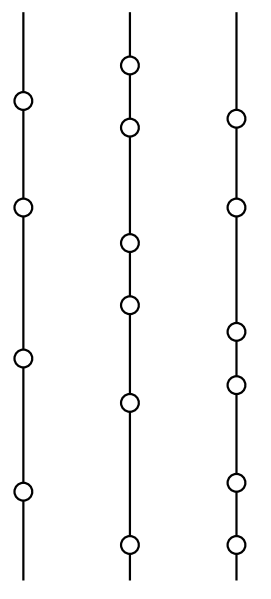

(a)

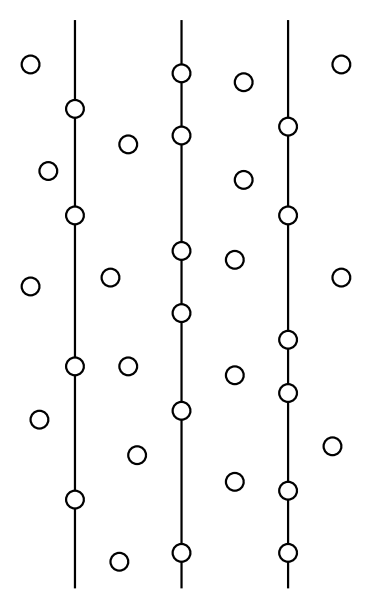

(b)

Fig. 2. Configurations of point disorder in (a) LD and (b) the periodic GL models. Figures are described in $x-y$ plane. Solid lines, in $x$ direction, denote the "superconducting layers", and the open circles denote the point defects for the pair-field. 


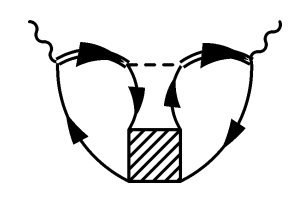

(a)
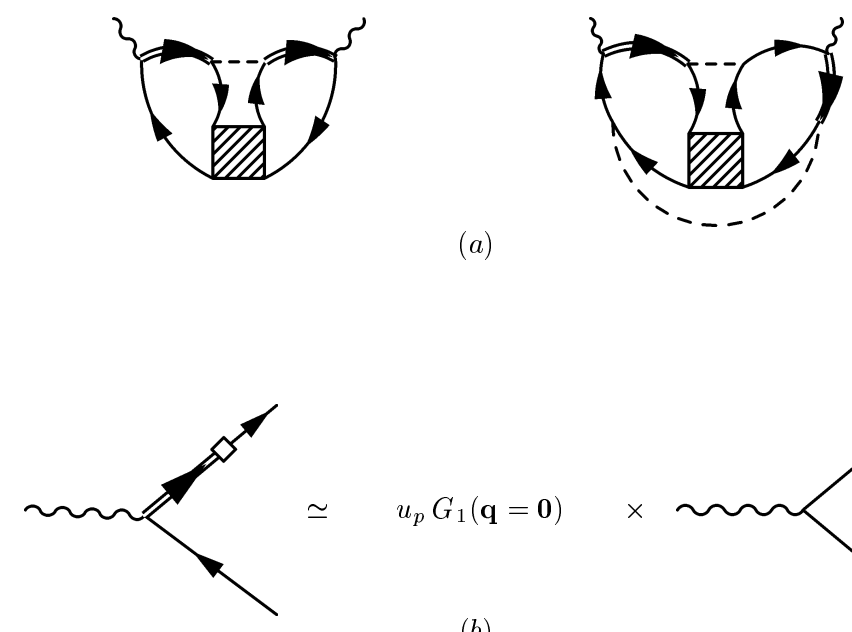

$u_{p} G_{1}(\mathbf{q}=\mathbf{0}) \quad \times$

(b)
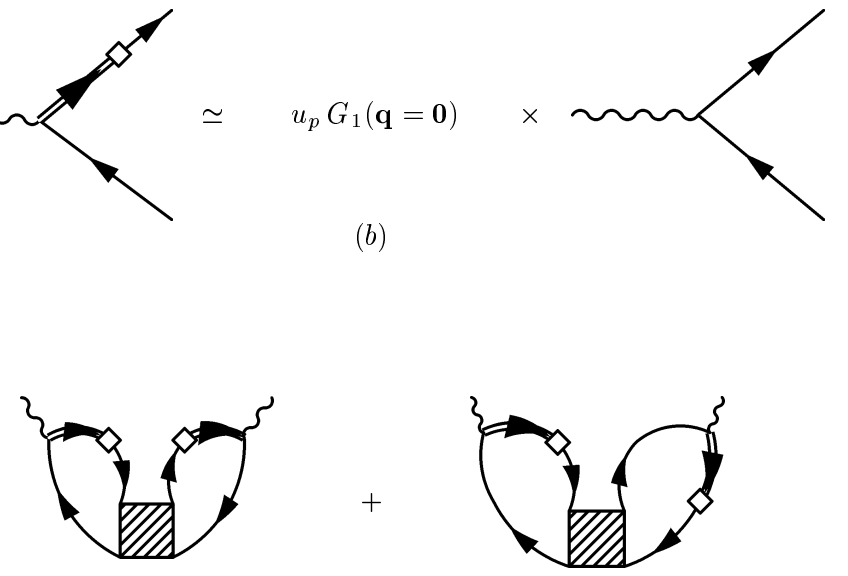

(c)

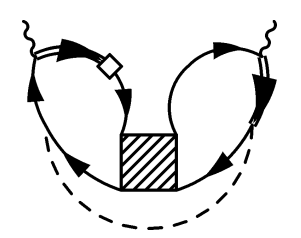

$(d)$

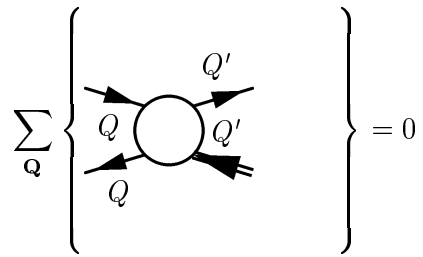

$(e)$

Fig. 3. Feynman diagrams on tilt moduli in the high field approximation. (4) The dashed line denotes the impurity line occuring after averaging over the point defect configurations and carries the factor $\Delta$, and the connection marked by an open square between a NLL propagator $G_{1}(\mathbf{q})$ (the double-solid line with arrow) and a LLL propagator (the solid line with arrow) implies the periodic potential with strength $u_{p}$. The hatched square and open sphere in (e) imply four-point vertices composed only of the LLL modes. See the text for further details. 


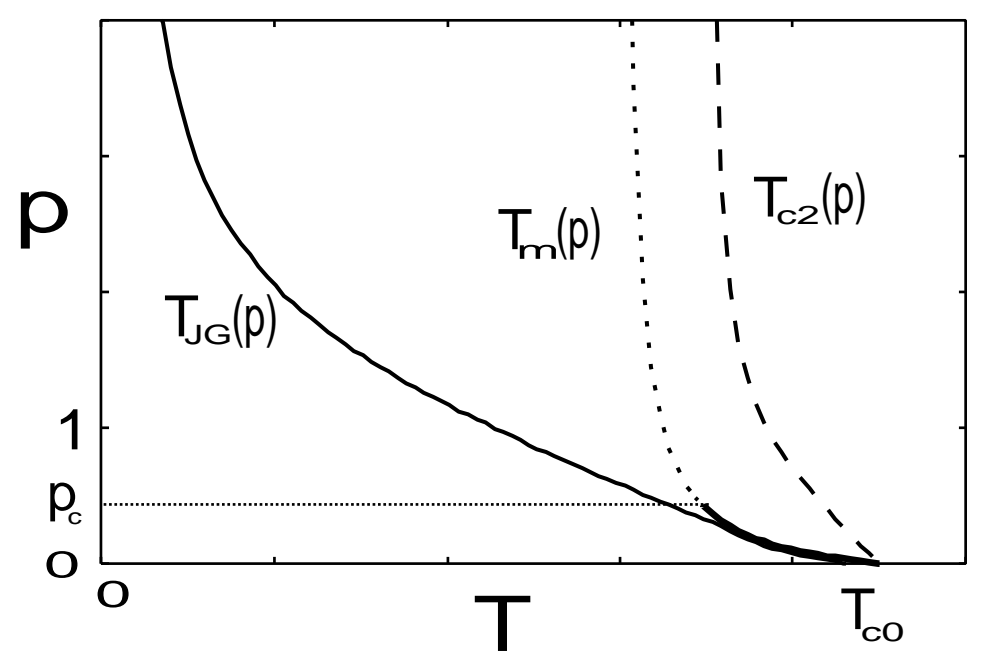

(a)

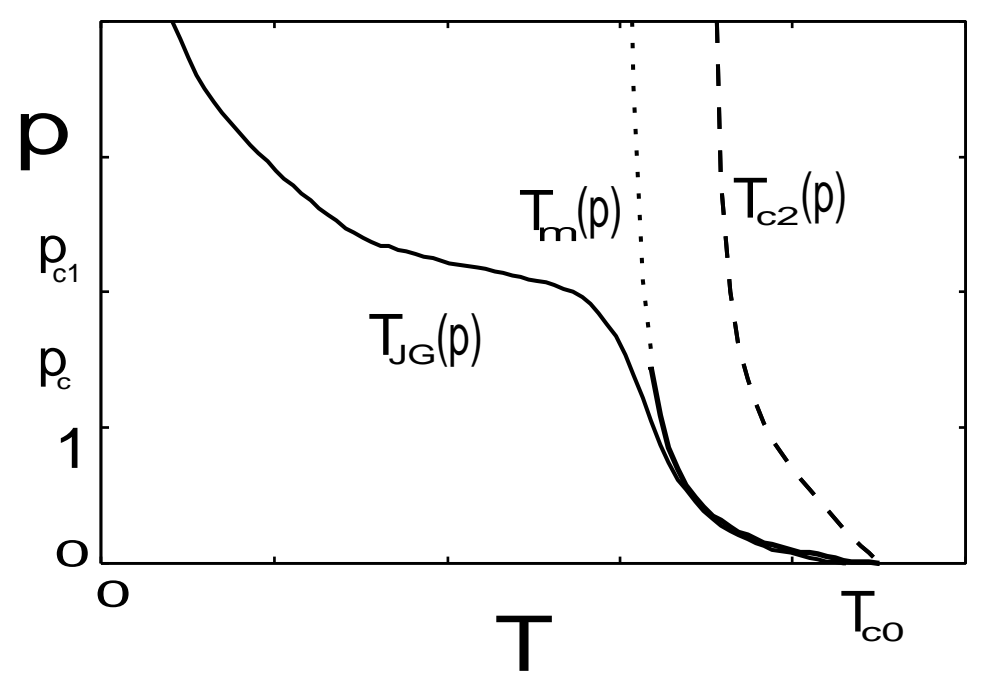

(b)

Fig. 4. Schematic $p$ v.s. $T$ phase diagrams of real layered superconductors in $\mathbf{B} \|$ layers for (a) dirtier and (b) cleaner cases resulting from the present study. The value $p_{c}$, the $p$ value at which the first order transition (expressed by the solid curve on $T_{m}(p)$ ) ends, measures the disorder strength. The relation (2.24) will be satisfied entirely in $p>p_{c}$ in the dirtier case (a), while it is valid only in $p>p_{c 1}\left(>p_{c}\right)$ in case (b). In each figure, the left solid curve $T_{J G}(p)$ is the JG transition line, while the right dashed curve $T_{c 2}(p)$ indicates the $H_{c 2}(T)$ curve. 